\title{
Toward global beyond-mean-field calculations of nuclear masses and low-energy spectra
}

\author{
Tomás R. Rodríguez, ${ }^{1,2}$ Alexander Arzhanov, ${ }^{2}$ and Gabriel Martínez-Pinedo ${ }^{2,3}$ \\ ${ }^{1}$ Departamento de Física Teórica, Universidad Autónoma de Madrid, E-28049 Madrid, Spain \\ ${ }^{2}$ Institut für Kernphysik, Technische Universität Darmstadt, D-64289 Darmstadt, Germany \\ ${ }^{3}$ GSI Helmholtzzentrum für Schwerionenforschung, Planckstraße 1, 64291 Darmstadt, Germany \\ (Received 29 July 2014; revised manuscript received 17 March 2015; published 20 April 2015)
}

\begin{abstract}
Self-consistent mean-field (MF) and beyond-mean-field (BMF) calculations of masses, separation energies, and $2_{1}^{+}$excitation energies of even-even nuclei where experimental data is available are presented. The functionals used are based on the Gogny D1S and D1M parametrizations and the method includes BMF corrections coming from both axial quadrupole shape mixing and symmetry restorations without assuming Gaussian overlap approximations. A comparison between MF and BMF approaches and the experimental data is provided. Additionally, the convergence of the results and the possible reduction of the magic shell gaps by including BMF effects are also discussed.
\end{abstract}

DOI: 10.1103/PhysRevC.91.044315

\section{INTRODUCTION}

Nuclear binding energies are one of the most relevant quantities that define the atomic nucleus. Apart from their intrinsic interest, the nuclear masses are especially important in determining the nucleosynthesis processes that occur in astrophysical environments. For example, they determine the limits of existence of the nucleus as a bound system of protons and neutrons (drip lines), the $Q$ values for $\beta$ and $\alpha$ decays, the particle separation energies that are needed to compute capture/emission rates, etc. Therefore, a great effort in measuring masses of very exotic (short-lived) nuclei with high precision is currently made with the use of trapping and/or storage rings techniques [1].

Despite this significant progress, and that expected in the near future with the arrival of new facilities worldwide, many nuclei will not be experimentally reachable. Particularly important are those belonging to neutron-rich regions that are relevant to better constrain the rapid neutron capture $(r$ process) nucleosynthesis, the mechanism behind the origin of more than the half of the elements beyond iron in the universe. Hence, nuclear models able to compute with high precision the known masses, as well as to provide reliable extrapolations, are very much demanded.

Current theoretical nuclear mass tables are provided mainly by three different approaches: (a) microscopicmacroscopic (mic-mac) methods which improve the original liquid drop formula with microscopic corrections (the most commonly used of this kind are the finite-range droplet model (FRDM) [2,3] or, more recently, the Weizsäcker-Skyrme (WS) mass model [4,5]); (b) Duflo-Zuker (DZ) approach based a functional of occupation numbers guided by the interacting shell model method [6]; (c) microscopic methods based on Hartree-Fock-Bogoliubov (HFB) approaches with Skyrme (see Refs. [7,8] and references therein) and Gogny functionals [9]. The above methods have reached a root mean square (rms) deviation from data $[10,11]$ of $0.57 \mathrm{MeV}$ (FRDM), $0.298 \mathrm{MeV}$ (WS), $0.36 \mathrm{MeV}$ (DZ), $0.51 \mathrm{MeV}$ (HFB-Skyrme), and $0.798 \mathrm{MeV}$ (HFB-Gogny).

This impressive agreement to the global behavior of the known masses is not sufficient to have a full confidence in the
PACS number(s): 21.10.Dr, 21.30.-x, 21.60.Jz, 21.60.Ev

extrapolations to unknown regions because large deviations in the predictions of the different models are found. In addition, even if the overall performance is similar, some local deviations between the models in sensitive regions can largely affect the results of nucleosynthesis simulations [12,13]. Therefore, theoretical models should be improved to reduce such uncertainties. In particular, $a b$ initio calculations are becoming available for medium mass systems although currently neither the range of applicability nor the accuracy reached by such methods are good enough to be applied to astrophysical purposes [14-20]. Hence, in the short-midterm energy density functionals are still the most promising microscopic methods to compute nuclear properties in the whole nuclear chart with the required accuracy. These methods have been improved significantly in recent years by taking into account not only global fits to all known masses but also some constraints coming from $a b$ initio calculations in infinite nuclear matter $[7,21,22]$.

However, a mean-field (MF) approach is commonly used to solve the nuclear many-body problem (HFB) and beyond-mean-field (BMF) corrections have been included phenomenologically to mimic rotational and vibrational corrections [23]. Nevertheless, in the past decade, a better treatment of such BMF correlations of even-even nuclei using symmetry restorations and configuration mixing methods have been implemented with Skyrme, relativistic, and Gogny interactions [24]. These improvements have allowed the study of the appearance/disappearance of shell closures or shape mixing/coexistence/transitions phenomena, for example. Concerning nuclear masses, these BMF correlations have been computed globally using particle-number and angular-momentum projection and generator coordinate method (GCM) with Skyrme interactions $[25,26]$ and using the five-dimensional collective Hamiltonian $(5 \mathrm{DCH})$ with Gogny [9,27] and, more recently, relativistic [28] interactions. Owing to the large computational burden of performing GCM with particle-number and angular-momentum restorations, these pioneer global surveys were carried out assuming Gaussian overlap approximations (GOAs).

In this paper we present the results of global calculations for even-even nuclei performed with the Gogny D1S [29,30] 
and D1M [9] interactions using implementations of the GCM and quantum number projection methods without GOAs. Additionally, we discuss the convergence of the results-both $\mathrm{HFB}$ and $\mathrm{BMF}$ - as a function of the number of oscillator shells included in the basis where the many-body wave functions are expanded.

Results for odd systems including such advanced manybody techniques are still not available with the Gogny interaction although some preliminary calculations in a single nucleus have been recently reported with Skyrme forces [31]. Additionally, in this survey we assume axial and parity symmetry conserving intrinsic states, exploring explicitly the axial quadrupole degree of freedom by performing onedimensional GCM calculations. Including triaxiality and/or octupolarity within the present framework increases prohibitively the computational time and it is beyond the scope of the present study. Nevertheless, global calculations including the triaxial $[9,27,32]$ and octupole $[33,34]$ degrees of freedom within less involved many-body methods have been reported. In particular, 159 of 5900 nuclei with triaxial ground states are found in the FRDM model [32]. The largest differences in binding energies, $\lesssim 0.6 \mathrm{MeV}$, are obtained in regions around ${ }^{108} \mathrm{Ru}$ and ${ }^{140} \mathrm{Gd}$. In Ref. [27] most of the nuclei are predicted to be axial symmetric (spherical, prolate, and oblate) at the HFB level with Gogny D1S. BMF correlations within the $5 \mathrm{DCH}$ bring all the nuclei toward triaxial shapes-on average - even though in the HFB approximation are predicted spherical and/or axial symmetric. On the other hand, following Ref. [34], only a few of the 818 nuclei calculated with Gogny D1S-D1N-D1M between $8 \leqslant Z \leqslant 110$ are octupole deformed in the HFB ground state. These nuclei are obtained around $\mathrm{Ra}, \mathrm{Ba}$, and $\mathrm{Zr}$ region and the energy gain with respect to the octupole symmetric states are $\lesssim 1.2 \mathrm{MeV}$. Further BMF correlations including parity projection and GCM along the $\beta_{3}$ degree of freedom give extra binding energies as large as $2.5 \mathrm{MeV}$ in the regions showing octupole deformation at the HFB level. However, in the rest of the nuclei these correlation energies are of the order of $1 \mathrm{MeV}$ and vary smoothly with $N$ and $Z$, producing an almost constant shift in the total energies.

This document is organized as follows. First, a description of the method used to compute masses is given in Sec. II. Then the results are discussed in Sec. III. Finally, a brief summary and outlook are presented in Sec. IV.

\section{THEORETICAL FRAMEWORK}

The total energy (negative in our convention) of a given nucleus is calculated in the present work as the sum of two terms,

$$
E=E_{\mathrm{HFB}}+\Delta E_{\mathrm{BMF}},
$$

where $E_{\mathrm{HFB}}$ is the $\mathrm{HFB}$ energy $(\mathrm{MF})$ and $\Delta E_{\mathrm{BMF}}$ is a $\mathrm{BMF}$ correction which includes particle-number restoration, angular-momentum projection, and axial quadrupole shape mixing within the GCM [35]. Both terms are computed with the same underlying interaction, namely, Gogny D1S or D1M parametrization.

For the sake of simplifying the notation, we express the theoretical energy throughout this work as the expectation value of a hamiltonian operator. However, it is important to point out that because the effective interactions used here are density-dependent, this notation is not truly rigorous and energy density functionals (EDFs) should be defined instead of such expectation values. We refer to Ref. [36] for a general discussion about this topic and Refs. [37,38] for the particular choice of the corresponding EDFs used in this work.

\section{A. Mean-field (HFB) approach}

We start by reviewing briefly the HFB method [35]. In this microscopic approach, based on the variational principle, the many-body wave function of the atomic nucleus is searched among a set of trial wave functions that are defined as quasiparticle vacua, $|\phi\rangle$ :

$$
\hat{\beta}_{k}|\phi\rangle=0 \forall k,
$$

Those quasiparticles are defined as the most general linear combination of creation $\left(\hat{c}_{l}^{\dagger}\right)$ and annihilation $\left(\hat{c}_{l}\right)$ singleparticle operators,

$$
\hat{\beta}_{k}^{\dagger}=\sum_{l} U_{l k} \hat{c}_{l}^{\dagger}+V_{l k} \hat{c}_{l},
$$

where $U$ and $V$ are the variational parameters. Because Eq. (3) breaks most of the symmetries of the original interaction, in particular, the particle-number symmetry, the HFB wave function is constrained to have the correct mean value of the number of particles. Therefore, the HFB equations [35] are found by the condition

$$
\delta\left(E_{\mathrm{HFB}}^{\prime}[|\phi\rangle]\right)_{|\phi\rangle=|\mathrm{HFB}\rangle}=0,
$$

with

$$
E_{\mathrm{HFB}}^{\prime}[|\phi\rangle]=\langle\phi|\hat{H}| \phi\rangle-\lambda_{N}\langle\phi|\hat{N}| \phi\rangle-\lambda_{Z}\langle\phi|\hat{Z}| \phi\rangle,
$$

where $\hat{H}$ and $\hat{N}(\hat{Z})$ are the Hamiltonian and the neutron (proton) number operators, respectively; $|\phi\rangle=|\mathrm{HFB}\rangle$ is the HFB solution obtained by solving the corresponding HFB equations [Eqs. (4)-(5)]; and $\lambda_{N(Z)}$ is a Lagrange multiplier which ensures $\langle\phi|\hat{N}(\hat{Z})| \phi\rangle=N(Z)$. The normalization $\langle\phi \mid \phi\rangle=1$ is also assumed.

In practical applications, the spherical harmonic oscillator (s.h.o.) basis is usually chosen as the working basis where the quasiparticles defined in Eq. (3) are expanded [39]. The sum in such an equation runs over an infinite number of s.h.o. states, but this sum must be truncated in actual implementations in computer codes. The results should not depend on the choice of the basis and the convergence of the results are obtained if a sufficiently large number of major s.h.o. shells $\left(N_{\text {s.h.o. }}\right)$ are included. However, the computational burden increases significantly with the number of oscillator shells and a compromise between a better convergence and a reasonable computational time has to be considered (see discussion below). In the present work, $E_{\mathrm{HFB}}=\langle\mathrm{HFB}|\hat{H}| \mathrm{HFB}\rangle$ in Eq. (1) is computed with $N_{\text {s.h.o. }}=19$.

\section{B. Beyond-mean-field approach}

The second term in the energy $\left[\Delta E_{\mathrm{BMF}}\right.$ in Eq. (1)] corresponds to corrections beyond the MF (HFB) approximation. 
In principle, the energy should be computed by using BMF methods from the first place. However, these methods are much more time consuming than the corresponding HFB and the size of the s.h.o. basis used in this case is generally smaller. In the present work, $N_{\text {s.h.o. }}=11$ has been chosen for computing BMF effects. Hence, BMF correction is defined as

$$
\Delta E_{\mathrm{BMF}}=E_{\mathrm{BMF}}\left(N_{\text {s.h.o. }}=11\right)-E_{\mathrm{HFB}}\left(N_{\text {s.h.o. }}=11\right) .
$$

This energy difference is less dependent on the number of oscillator shells included in the basis than the total energies separately, $E_{\mathrm{HFB}}$ and $E_{\mathrm{BMF}}$. We study explicitly this point below.

In the above equation, $E_{\mathrm{BMF}}\left(N_{\text {s.h.o. }}=11\right)$ is computed within a symmetry-conserving configuration mixing (SCCM) framework. The method contains simultaneous particlenumber and angular-momentum projection (PNAMP) of different intrinsic HFB-type states and a subsequent mixing of these states performed within the GCM framework. In general, different shapes (axial and nonaxial) and collective coordinates can be included in the GCM calculation. However, the addition of more generating coordinates largely increases the computational time, especially if a triaxial angular momentum projection is performed. Therefore, we have focused on the mixing of axial deformed and parity-conserving states. Further extensions will be explored in a future study. Nevertheless, contrary to other BMF approaches like the $5 \mathrm{DCH}$, this method is variationally consistent with the underlying HFB functional; i.e., lower total energies are always obtained when such correlations are taken into account. Furthermore, the more collective coordinates and symmetry restorations are included in the GCM the lower is the ground-state energy obtained until the exact solution is eventually obtained. In this sense, the method is extensible to other degrees of freedom (triaxiality, octupolarity, pairing content,...) without losing its variational character. Finally, although the present calculations are restricted to axial, parity, and time-reversal symmetric intrinsic states, the method can be applied to the whole nuclear chart. If a specific nucleus turns out to be, for instance, triaxial and/or octupole deformed, the amount of correlations obtained here will be smaller, though neither negligible nor meaningless, than the ones eventually obtained with including those degrees of freedom.

In the following, the particular realization of the SCCM method used here is described step by step.

\section{Variation after particle-number projection method}

In contrast to the previous section (Sec. II A), the particle number projected energy is minimized instead of the HFB one. This is the so-called variation after particle-number projection (PN-VAP) [35,40]. Furthermore, the set of intrinsic, HFB-type, trial wave functions, $\left|\phi\left(\beta_{2}\right)\right\rangle$, are also constrained to have a given value of the axial quadrupole deformation, $\beta_{2}$. Hence, the variational equation now reads as

$$
\delta\left(E_{\mathrm{PN}-\mathrm{VAP}}^{\prime}[|\phi\rangle]\right)_{|\phi\rangle=|\mathrm{PN}-\mathrm{VAP}\rangle}=0,
$$

where $\left|\phi\left(\beta_{2}\right)\right\rangle=\left|\operatorname{PN}-\operatorname{VAP}\left(\beta_{2}\right)\right\rangle$ is the intrinsic wave function that minimizes the functional:

$$
E_{\mathrm{PN}-\mathrm{VAP}}^{\prime}[|\phi\rangle]=\frac{\left\langle\phi\left|\hat{H} P^{N} P^{Z}\right| \phi\right\rangle}{\left\langle\phi\left|P^{N} P^{Z}\right| \phi\right\rangle}-\lambda_{q_{20}}\left\langle\phi\left|\hat{Q}_{20}\right| \phi\right\rangle
$$

Here $P^{N(Z)}$ is the projector onto good number of neutrons (protons) [35], $\hat{Q}_{20}=r^{2} Y_{20}(\theta, \varphi)$ is the axial quadrupole operator, and the Lagrange multiplier $\lambda_{q_{20}}$ ensures the condition $\lambda_{q_{20}} \rightarrow\left\langle\phi\left|\hat{Q}_{20}\right| \phi\right\rangle=q_{20}$. The quadrupole deformation parameter $\beta_{2}$ is related to $q_{20}$ by

$$
q_{20}= \pm \frac{\beta_{2}}{C}, \quad C=\sqrt{\frac{5}{4 \pi}} \frac{4 \pi}{3 r_{0}^{2} A^{5 / 3}},
$$

where $r_{0}=1.2 \mathrm{fm}, A$ is the mass number, and the plus (minus) sign indicates prolate (oblate) shapes. Hence, the collective intrinsic deformation is well established within this framework and this fact allows the description of the states in the laboratory frame in terms of their intrinsic shapes unambiguously.

\section{Symmetry-conserving configuration mixing method}

Once the set of intrinsic wave functions- $\left|\mathrm{PN}-\operatorname{VAP}\left(\beta_{2}\right)\right\rangle-$ is obtained, the final states are built through the ansatz provided by the GCM [35]. In this framework, the states are assumed to be linear combinations of particle-number and angular-momentum projected PN-VAP states,

$$
\left|\Psi^{I \sigma}\right\rangle=\sum_{\beta_{2}} g_{\beta_{2}}^{I \sigma} P_{00}^{I} P^{N} P^{Z}\left|\operatorname{PN}-\operatorname{VAP}\left(\beta_{2}\right)\right\rangle,
$$

where $I$ is the total angular momentum, $P_{K K^{\prime}}^{I}$ is the angular momentum projector applied to axial symmetric intrinsic states $\left(K=K^{\prime}=0\right)$ [35], and $\sigma$ labels different states obtained for a given value of $I$. The parameters $g_{\beta_{2}}^{I \sigma}$ are determined by the Ritz variational principle which leads to the Hill-Wheeler-Griffin (HWG) equation:

$$
\begin{aligned}
\delta\left(E_{\mathrm{SCCM}}^{I \sigma}\left[g_{\beta_{2}}^{I \sigma}\right]\right) & =0 \Rightarrow \sum_{\beta_{2}^{\prime}}\left(\mathcal{H}_{\beta_{2}, \beta_{2}^{\prime}}^{I}-E_{\mathrm{SCCM}}^{I \sigma} \mathcal{N}_{\beta_{2}, \beta_{2}^{\prime}}^{I}\right) g_{\beta_{2}^{\prime}}^{I \sigma} \\
& =0 .
\end{aligned}
$$

The energy and norm overlap matrices are defined as

$$
\begin{aligned}
\mathcal{H}_{\beta_{2}, \beta_{2}^{\prime}}^{I} & =\left\langle\operatorname{PN}-\operatorname{VAP}\left(\beta_{2}\right)\left|\hat{H} P_{00}^{I} P^{N} P^{Z}\right| \operatorname{PN}-\operatorname{VAP}\left(\beta_{2}^{\prime}\right)\right\rangle, \\
\mathcal{N}_{\beta_{2}, \beta_{2}^{\prime}}^{I} & =\left\langle\operatorname{PN}-\operatorname{VAP}\left(\beta_{2}\right)\left|P_{00}^{I} P^{N} P^{Z}\right| \operatorname{PN}-\operatorname{VAP}\left(\beta_{2}^{\prime}\right)\right\rangle .
\end{aligned}
$$

The resulting HWG equations-one for each value of the angular momentum-provide the energy levels $E_{\mathrm{SCCM}}^{I \sigma}$ and collective wave functions defined in the $\left(\beta_{2}\right)$ direction.

Hence, the energy including symmetry restorations and shape mixing within this framework is given by

$$
E_{\mathrm{BMF}}\left(N_{\text {s.h.o. }}=11\right)=E_{\mathrm{SCCM}}^{I=0 \sigma=1} .
$$


Obviously, excited states, in particular $E\left(2_{1}^{+}\right)=E_{\mathrm{SCCM}}^{I=2 \sigma=1}-$ $E_{\mathrm{SCCM}}^{I=0 \sigma=1}$ excitation energies, can be also calculated within the same framework.

\section{Numerical details and convergence of the method}

We summarize some details about the actual global calculations. First of all, the HFB [41], PN-VAP [40], and SCCM [42] codes used throughout this work have been developed in the Nuclear Physics Group at the Universidad Autónoma de Madrid. The calculations were performed both in GSIPrometheus (Darmstadt) [43] and CSC-Loewe (Frankfurt) [44] computing facilities, using scripts optimized to perform such a large-scale survey. The HFB (MF) solutions have been found by using a s.h.o. basis with $N_{\text {s.h.o. }}=19$ shells and an optimized oscillator length for each nucleus [45]. Additionally, with this large number of s.h.o. shells, potential energy surfaces along the axial quadrupole degree of freedom were explored to make sure that the unconstrained HFB calculations did not converge to local metastable minima. All terms (direct, exchange, and pairing) in the interaction (including Coulomb) have been included here and also in the BMF part. For the GCM part, a set of 15-20 intrinsic many-body wave functions with different axial quadrupole shapes (oblate and prolate) has been found by using the PN-VAP method described above. These intrinsic wave functions are expanded in a basis with $N_{\text {s.h.o. }}=11$ shells, again with an optimized oscillator length for each nucleus. The standard number of points used in the integrals in the gauge (particle number projection) and Euler (angular momentum projection) angles were 9 and 16, respectively, and the convergence of the quantum number projections were checked by inspecting the mean values of the operators, $\hat{N}, \hat{Z}, \Delta \hat{N}^{2}, \Delta \hat{Z}^{2}$, and $\hat{J}^{2}$.

Finally, the convergence of the solutions of the HWG equations has been ensured by analyzing the energy plateaus as a function of the natural states which transform the HWG equations into regular eigenvalue problems. Detailed expressions and performance of this approach can be found in Refs. [38,42] (and references therein).

To shed light on how the BMF method used here actually works, the nucleus ${ }^{120} \mathrm{Cd}$ is taken as an example. The HFBGogny D1S-energy calculated with $N_{\text {s.h.o. }}=19$ is $E_{\mathrm{HFB}}=$ $-1011.786 \mathrm{MeV}$. On top of this value, BMF corrections are made [see Eq. (1)]. As mentioned in the previous section, these corrections are performed with $N_{\text {s.h.o. }}=11$. In Fig. 1 the energy as a function of the axial quadrupole deformation $\beta_{2}$ is represented for MF (HFB, dotted line), variation after particle number projection (PN-VAP, dashed line), and particle-number and angular-momentum $I=0$ projection (PNAMP, $I=0$, thin continuous line) approximations. The minima of each potential energy surfaces are the corresponding energies for each level of approximation (square, HFB; triangle PN-VAP; and diamond, PNAMP, $I=0)$. In this case, the value of deformation of all the minima is quite similar ( $\left.\beta_{2} \sim 0.17\right)$, i.e., ${ }^{120} \mathrm{Cd}$ is prolate deformed in all of these approaches. A gain in the energy $(1.96 \mathrm{MeV})$ is observed when correlations associated to the restoration of the particle number are taken into account. Further correlation energy $(2.80 \mathrm{MeV})$ is obtained when simultaneous particle-number

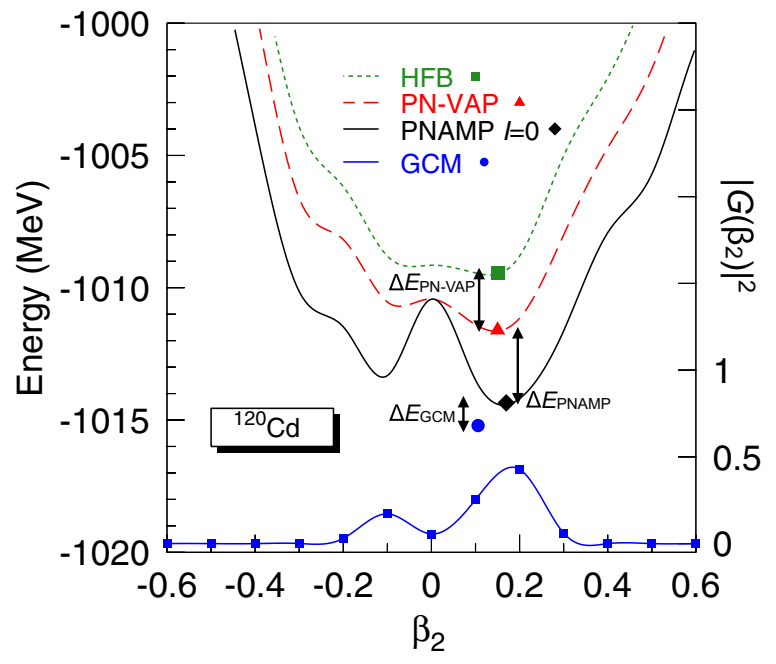

FIG. 1. (Color online) Potential energy surfaces as a function of the axial quadrupole deformation calculated with HFB (green dotted line), PN-VAP (red dashed line), and PNAMP (thin black continuous line) approximations for ${ }^{120} \mathrm{Cd}$ with the Gogny D1S parametrization. The square, triangle, and diamond represent the minima of each surface. The blue dot corresponds to the full SCCM energy and the blue boxes (connected by a continuous line to guide the eye) represent the ground-state collective wave function normalize to 1 $\left(\sum_{\beta_{2}}\left|G\left(\beta_{2}\right)\right|^{2}=1\right)$. The arrows point out the energy gain between the different approaches considered in this work.

and angular-momentum projection is performed. In addition, by allowing shape mixing of particle-number and angularmomentum restored axial states (GCM), Eqs. (10) and (11), the energy marked by a blue dot in Fig. 1 is obtained, i.e., $1.02 \mathrm{MeV}$ extra energy with respect to the PNAMP $(I=0)$ minimum. The square of so-called ground-state collective wave function $\left[\left|G\left(\beta_{2}\right)\right|^{2}\right]$ is plotted and it represents the probability of having a given $\beta_{2}$ deformation in this state (blue boxes in Fig. 1). In this case, two maxima are found at $\beta_{2} \sim-0.1$ and +0.2 , being the prolate one the absolute maximum. The position of the blue dot in the abscissa axis corresponds to the mean deformation calculated with the ground-state collective wave function $\bar{\beta}_{2}=0.10$. In summary, the total correction provided by the current SCCM method is $\triangle E_{\mathrm{BMF}}=5.77 \mathrm{MeV}$ and the total energy [Eq. (1)] is $E\left({ }^{120} \mathrm{Cd}\right)=-1017.559 \mathrm{MeV}$.

We discuss next the performance and convergence of the results as a function of the number of s.h.o. shells, $N_{\text {s.h.o. }}$, included in the working basis. Because the results should not depend on the size of such a basis if a sufficient large number of single-particle states are included, in the ideal situation one should take a very large number for $N_{\text {s.h.o. }}$. However, this number is limited by the present computational resources. Thus, the average computing time required at each step of the calculation of one nucleus in a single core, depending on $N_{\text {s.h.o., }}$ is shown in Table I. Here we observe the huge differences in the computational burden between the different approaches, in particular when we compare the values needed for HFB and BMF methods. It is important to point out that while the running time for the GCM part can be established beforehand once the number of shells and GCM points are chosen, for the 
TABLE I. Estimation of the computational time used to calculate one nucleus in one single core at the GSI-Prometheus cluster for each level of approximation described in Sec. II. Shown in boldface is the number of shells chosen in global calculations.

\begin{tabular}{lccc}
\hline \hline$N_{\text {s.h.o. }}$ & HFB (h) & PN-VAP (h) & GCM (h) \\
\hline 11 & $\sim 0.07$ & $\sim \mathbf{4 5}$ & $\mathbf{1 4 . 5}$ \\
13 & $\sim 0.20$ & $\sim 120$ & 54.4 \\
15 & $\sim 0.52$ & $\sim 300$ & 169.1 \\
17 & $\sim 0.97$ & $\sim 500$ & 460.3 \\
19 & $\sim \mathbf{2 . 4 6}$ & - & - \\
\hline \hline
\end{tabular}

HFB part, and more critically, for the PN-VAP, those numbers can vary from nucleus to nucleus depending on the rate of convergence of the minimization process [Eqs. (4) and (7)]. Therefore, the values in Table I refer to average numbers in those cases. We can directly check in Table I that enlarging the number of shells for BMF calculations would increase prohibitively the computational time. In fact, we have chosen $N_{\text {s.h.o. }}=17$ as our current limit for BMF calculations.

In Fig. 2 we represent the dependence of the total energy, calculated with the different approaches, on the number of oscillator shells for a medium mass nucleus $\left({ }^{120} \mathrm{Cd}\right.$ mentioned above) and a heavy one $\left({ }^{194} \mathrm{Po}\right)$. In both cases we observe an energy gain when increasing $N_{\text {s.h.o. }}$ but only for ${ }^{120} \mathrm{Cd}$ is a convergence regime reached for the HFB result [Fig. 2(a)]. For the heavy nucleus ${ }^{194}$ Po [Fig. 2(b)], further energy gain is expected if more single-particle states are added to the working basis and $N_{\text {s.h.o. }}=19$ is not a converged value. Therefore, extrapolation methods to an infinite basis should be applied to further converge the total energy [9,46-51]. The performance and reliability of those extrapolation schemes within the present theoretical framework is a work in progress [52] and we have taken the value with $N_{\text {s.h.o. }}=19$ as our best converged one for the HFB result.

As a matter of fact, in Refs. [27,47] the HFB energy for Gogny D1S has been computed choosing a number of singleparticle states equal to eight times the larger number among the protons and neutrons in the nucleus. We have checked this prescription by comparing the Gogny-D1S-HFB values given in the supplemental material of Ref. [27] with our $N_{\text {s.h.o. }}=$ 19 , finding a systematic better convergence in our case of
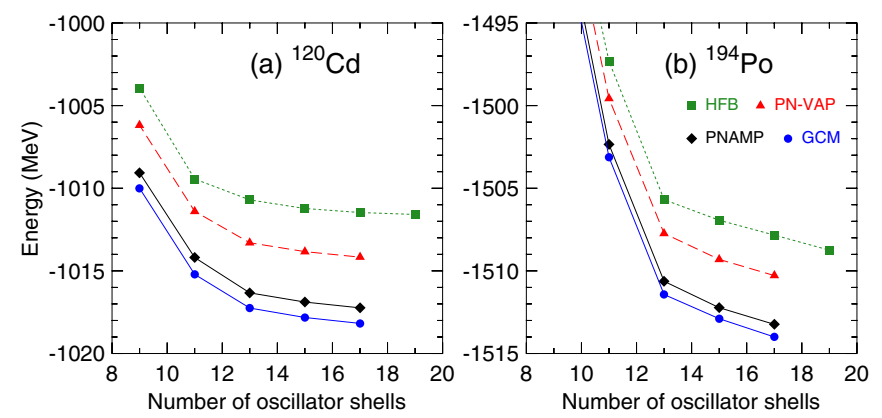

FIG. 2. (Color online) Convergence of the energy as a function of the number of major oscillator shells included in the working basis for the same approaches of Fig. 1. The left and right panels correspond to ${ }^{120} \mathrm{Cd}$ and ${ }^{194} \mathrm{Po}$, respectively, calculated with Gogny D1S.

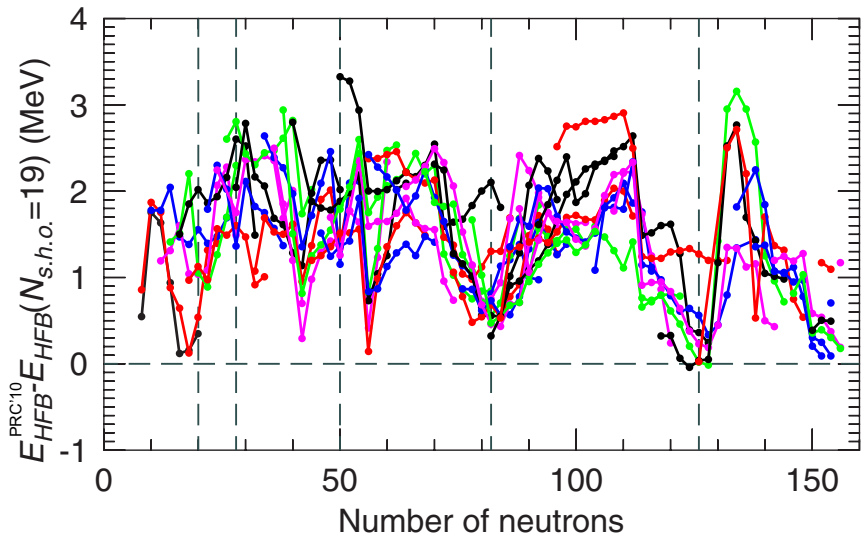

FIG. 3. (Color online) Differences between the HFB total energies given in Ref. [27] and the present HFB calculation performed with $N_{\text {s.h.o. }}=19$ and optimized oscillator length. Isotopic chains are connected by lines with the same color and the interaction in all of the cases is Gogny D1S. Positive values mean lower total energies obtained with the present calculation with respect to Ref. [27].

roughly $1.5 \mathrm{MeV}$ in the whole nuclear chart (see Fig. 3). These results point out that convergence of the total energy by using harmonic oscillator bases will be a possible source of problems of the present work which is also shared by previous calculations.

On the other hand, the total energies obtained with PN-VAP, PNAMP, and GCM approaches as a function of $N_{\text {s.h.o. }}$ are almost parallel to each other, showing that the BMF correlations, $\Delta E_{\mathrm{BMF}}$, are less dependent on the number of singleparticle states included in the basis (Fig. 2). Furthermore, as a consequence of the variational nature of our BMF correlations, larger correlations are obtained with the GCM method than the ones given by PNAMP, being the latter larger than PN-VAP as well.

As mentioned above, using Eq. (1) to compute the total energy emerges from the present limitations in terms of both convergence and computational time. Let us check whether the total energy computed with the SCCM method with a large number of harmonic oscillator shells $-N_{\text {s.h.o. }}=17$ is our limit—can be reproduced with the Eq. (1), computing the HFB part with such a large number of $N_{\text {s.h.o. }}$ and $\Delta E_{\mathrm{BMF}}$ with the significantly less time consuming $N_{\text {s.h.o. }}=11$ value.

In Fig. 4 we show the shell effects computed both with HFB and GCM methods for $Z=80,82$, and 84 isotopic chains. Shell effects are defined as the difference between the total (experimental or theoretical) energy and the energy provided by a liquid drop formula $\left(E_{\mathrm{LD}}\right)$ and it is a convenient way to rescale the total energy:

$$
\text { S.E. }(Z, N)=E_{\mathrm{LD}}(Z, N)-E(Z, N) .
$$

We observe first a large difference (up to $\sim 10 \mathrm{MeV}$ ) between the shell effects calculated with 11 and 17 oscillator shells within the same many-body approach, HFB or GCM, for all isotopic chains. This shows again explicitly that the total energy calculated with $N_{\text {s.h.o. }}=11$ is not well converged, now in three different isotopic chains. However, we can approach the BMF results with $N_{\text {s.h.o. }}=17$ by adding to the HFB values computed with the same number of oscillator shells, 

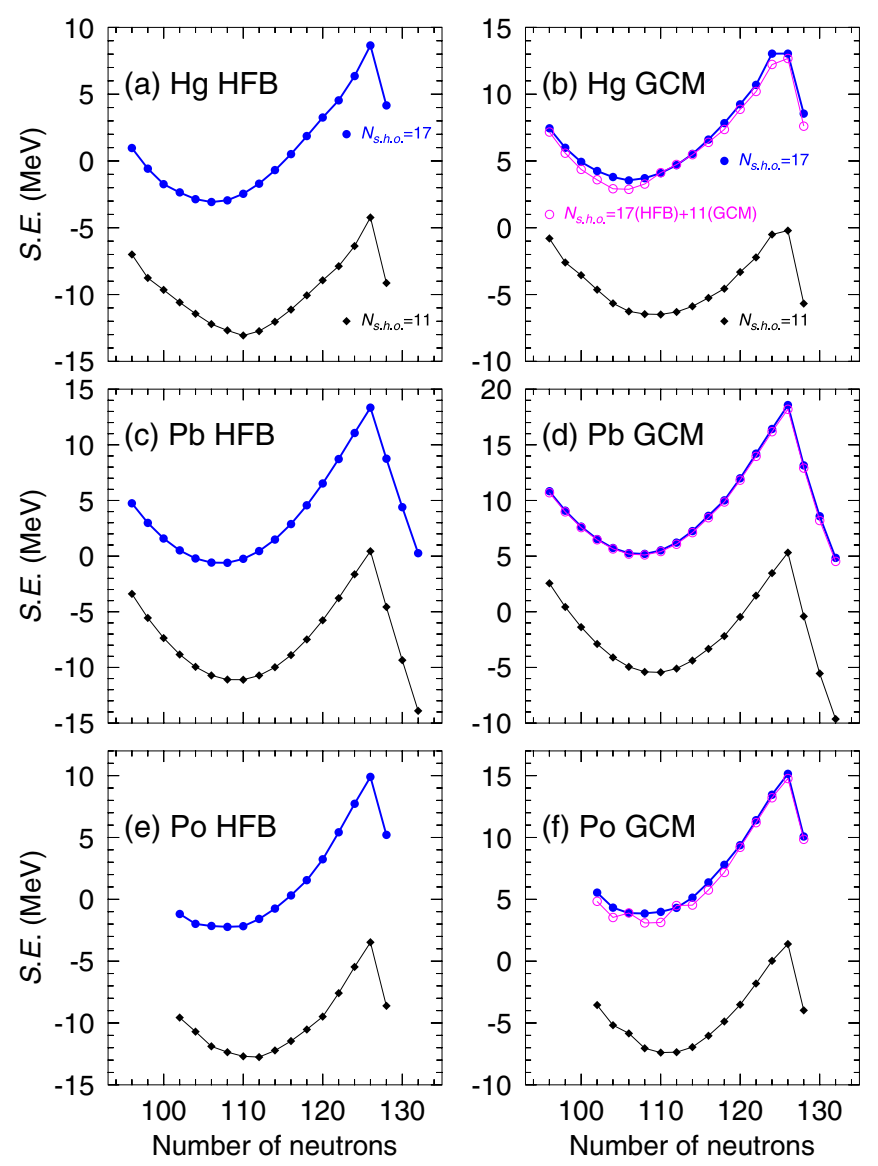

FIG. 4. (Color online) Shell effects [Eq. (14)] calculated with the HFB (left column) and GCM (right column) methods for $\mathrm{Hg}$ (top), $\mathrm{Pb}$ (middle), and Po (bottom) isotopic chains. Black diamonds and blue dots are calculated with $N_{\text {s.h.o. }}=11$ and 17, respectively. Magenta circles are calculated by adding the BMF corrections computed with $N_{\text {s.h.o. }}=11$ to the HFB result with $N_{\text {s.h.o. }}=17$. All nuclei are computed with the Gogny D1S parametrization.

the BMF corrections obtained with $N_{\text {s.h.o. }}=11$. Such a result is represented with magenta circles in Figs. 4(b), 4(d), and 4(f), showing a nice agreement with the GCM values calculated the largest value of $N_{\text {s.h.o. }}$ that we can reach for this approach.

Once we have checked the suitability of splitting the total energy in a MF part plus BMF corrections, we have performed additional tests of the convergence of the results in heavy nuclei. Hence, $Z=80-84$ isotopic chains and $N=124-128$ isotonic chains have been calculated in all of the approaches with increasing values of the oscillator shells (with the Gogny D1S parametrization). The results are summarized in Fig. 5, where shell effects (top row), BMF corrections (second row), two-neutron separation energies (third row), and $2^{+}$excitation energies (bottom row) are shown. In the top row of Fig. 5 the shell effects corresponding to HFB calculations are represented, although similar patterns, but shifted to larger values, are found in PN-VAP and GCM results (see right column of Fig. 4). We observe that, increasing $N_{\text {s.h.o. }}$, the results tend to collapse to a final curve. However, the differences between the HFB results for $N_{\text {s.h.o. }}=19$ and 17 are around $\sim 0.8 \mathrm{MeV}$ on average and one should go to a larger number of oscillator shells to get full convergence.

The large differences found in the shell effects for the $N_{\text {s.h.o. }}=11$ and $N_{\text {s.h.o. }}=19$ results (up to $14 \mathrm{MeV}$ ) are much smaller in the calculated BMF corrections. Furthermore, they are almost negligible in the two-neutron (-proton) separation energies, defined in Eq. (15). However, we also observe some local deviations from the $N_{\text {s.h.o. }}=17$ - chosen to be the best values for BMF approaches-in the neutron deficient $\mathrm{Hg}, \mathrm{Pb}$, and $\mathrm{Po}$ isotopes. In this region, several deformed configurations are almost degenerated (see Ref. [53] and references therein) that can be favored differently depending on the number of oscillator shells. Hence, those small jumps are produced by a change in deformation. In any case, the largest differences are around $0.9 \mathrm{MeV}$ but still could lead to small artificial jumps in $S_{2 n(2 p)}$ and/or $E\left(2_{1}^{+}\right)$. The above aspects are also visualized in the corresponding ratios between the results with $N_{\text {s.h.o. }}=17$ and $N_{\text {s.h.o. }}=11$ represented in the insets of Fig. 5. Therefore, these convergence effects should be taken into account for improving the precision of the mass models.

Finally, the comparison with the available experimental data for even-even nuclei requires the calculation of 598 nuclei for each parametrization of the Gogny functional, i.e., 1196 nuclei. Therefore, as mentioned in previous sections, the present global survey has been restricted to compute the HFB energy and the BMF corrections using $N_{\text {s.h.o. }}=19$ and 11, respectively. According to the previous analysis, this choice seems to be a reasonable compromise between convergence and computational time. This means that the calculation of one nucleus within the prescription followed in this work takes $\sim 62 \mathrm{~h} /$ core at the GSI-Prometheus cluster, which is still feasible with our current facilities.

\section{RESULTS}

\section{A. BMF correlation energies}

We now generalize the results obtained in Fig. 1 to the even-even nuclei with $Z, N \geqslant 10$ contained in the most recent atomic mass evaluation (AME) [11] both for Gogny D1S and D1M parametrizations. In Fig. 6 the successive gains in total energy reached by restoring the symmetries and allowing the axial quadrupole shape mixing are represented as a function of the number of neutrons. The first noticeable aspect is the striking similarity between those correlation energies for both D1S and D1M parametrizations. We observe a band of values ranged in the interval $1.5-3.5 \mathrm{MeV}$ with a mean gain $\sim 2.3 \mathrm{MeV}$ of correlation energy with respect to the MF (HFB) solutions when variation after particle-number projection (PN-VAP) method is applied [Figs. 6(a)-6(b)]. In addition, some local minima are obtained around $\sim 24, \sim 44, \sim 78$, and $\sim 110$, right before the neutron magic numbers $28,50,82$, and 126 for both D1S and D1M parametrizations.

Minima are also found both in the energy gained by particlenumber and angular-momentum restoration (PNAMP)—on top of PN-VAP — and by the GCM - on top of PNAMP — but now located at the shell closures. In the former [Figs. 6(c) and 6(d)], larger correlation energies are obtained in the 

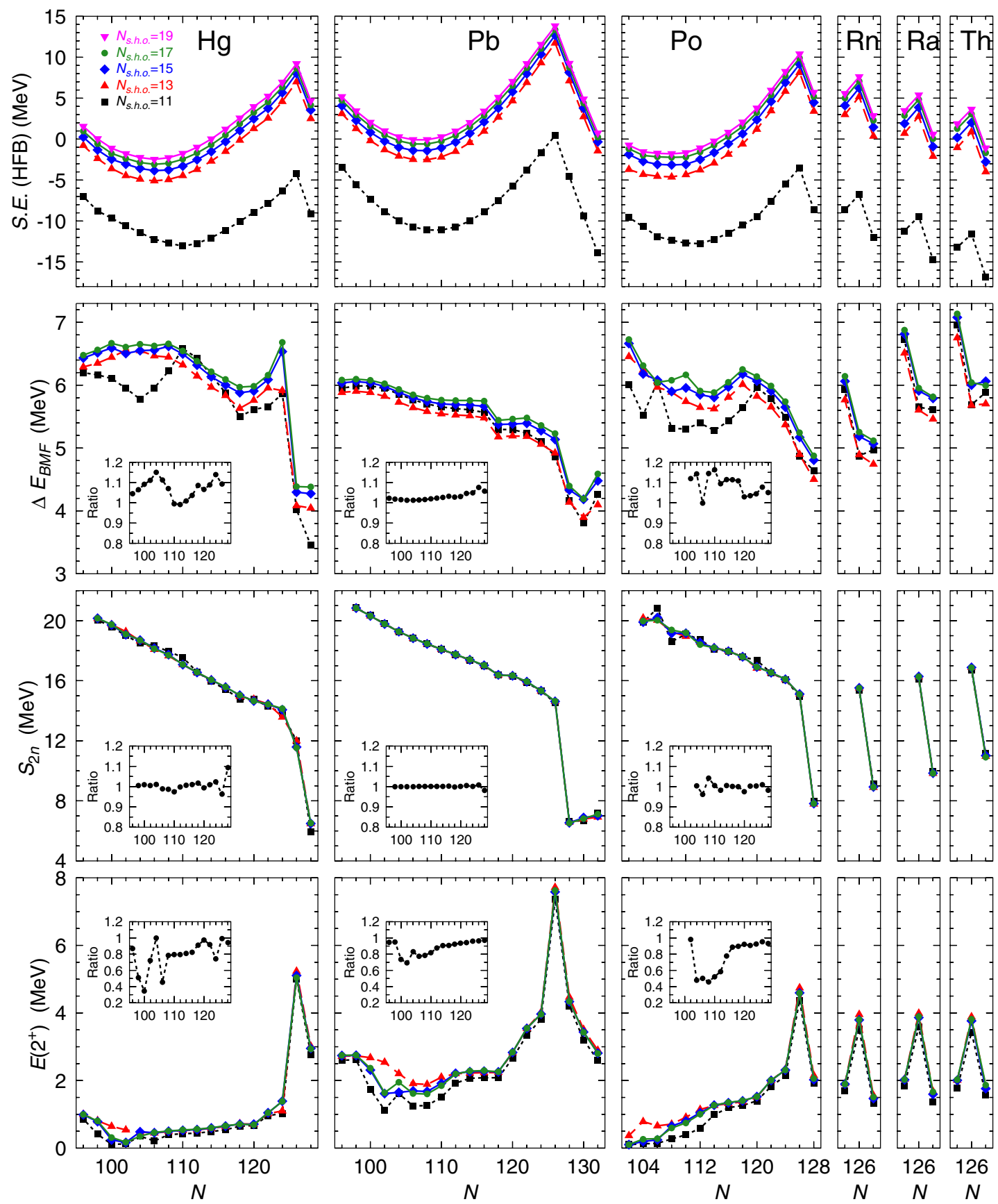

FIG. 5. (Color online) Results of the calculations performed with different values of $N_{\text {s.h.o. }}$ for $\mathrm{Hg}$, $\mathrm{Pb}$, Po, Rn, Ra, and Th isotopes (from left to right). (Top row) Shell effects calculated with the HFB method; (second row) BMF corrections; (third row) two-neutron separation energies with BMF corrections; and (bottom row) $2_{1}^{+}$excitation energies. In the insets, the ratios between the results with $N_{\text {s.h.o. }}=17$ and 11 are represented. All nuclei are computed with the Gogny D1S parametrization.

midshell and minima are found at the neutron magic numbers. Excluding the lighter and the semimagic nuclei, an average gain of $\sim 2.7 \mathrm{MeV}$ is attained and a slightly decreasing slope is also observed when increasing the number of neutrons. Concerning the GCM correlation energies [Figs. 6(e) and 6(f)], contrary to PNAMP, the larger gains are almost at the shell closures, obtaining a clear maximum at $N=126$. The average gain in this case is $\sim 0.8 \mathrm{MeV}$. This behavior of the BMF energies is important to correct the parabolic shape observed in experimental theoretical energy differences at the HFB level (see Fig. 10).

Finally, the total BMF energy gain (summing up all of the previous contributions) is represented in Figs. 6(g) and 6(h). We observe first that smaller correlation energies are obtained around the magic numbers, producing qualitatively an inverted parabolic behavior between two consecutive shell closures. Furthermore, we see a larger spread in the BMF energy gain in the lighter nuclei. Finally, the overall gain is slightly 

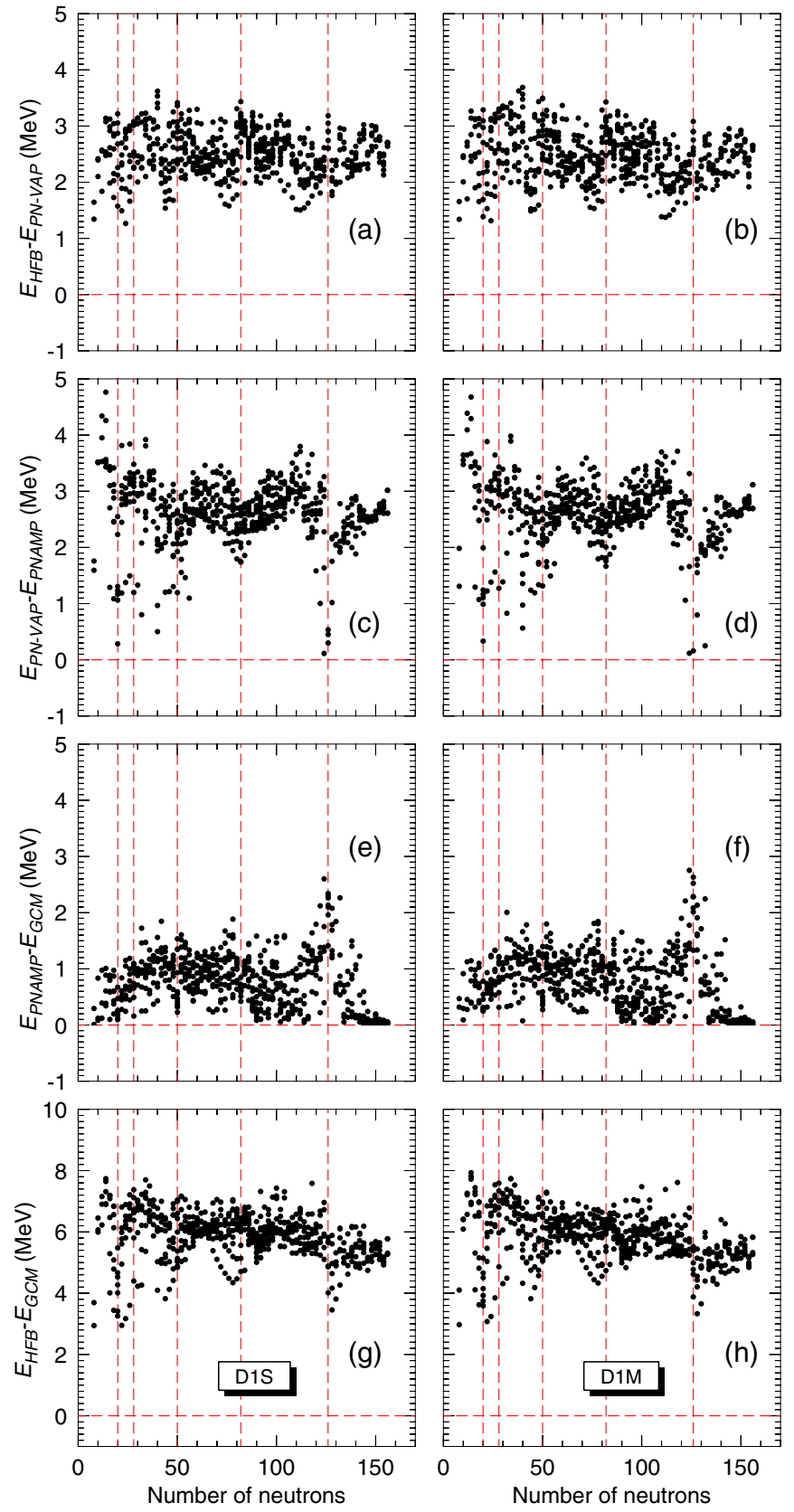

FIG. 6. (Color online) Gain in total energy as a function of the number of neutrons obtained by including (a),(b) variation after particle-number projection (PN-VAP) method; (c),(d) simultaneous particle-number and angular-momentum $I=0$ projection (PNAMP); (e),(f) quadrupole shape mixing within the GCM with symmetry restored states; and $(\mathrm{g}),(\mathrm{h})$ total BMF gain. Dashed vertical lines represent the neutron magic numbers 20, 28, 50, 82, and 126. Left and right columns correspond to Gogny D1S and D1M parametrizations, respectively.

smaller when the number of neutrons is increased for both parametrizations.

Patterns analogous to the ones just described above are found for the different levels of approximation when they are represented as a function of the number of protons (see Fig. 7). Hence, a rather flat energy gain for the PN-VAP
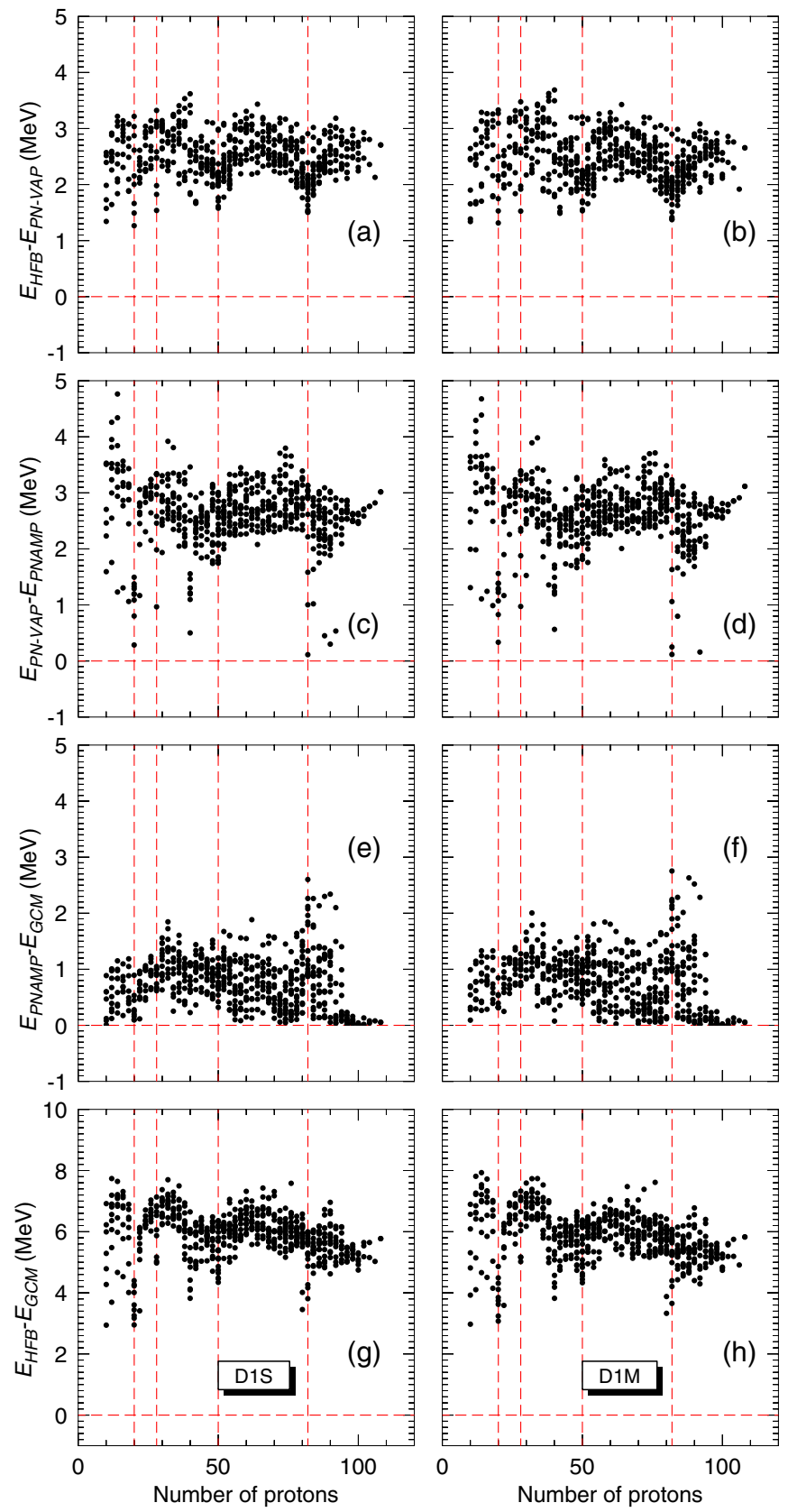

FIG. 7. (Color online) Same as Fig. 6 but as a function of the number of protons. Dashed vertical lines represent the proton magic numbers $20,28,50$, and 82 .

approach is obtained both for D1S and D1M parametrizations [Figs. 7(a) and 7(b)]. Furthermore, the local minima are found now at the proton magic numbers $20,28,50$, and 82 in this approximation. For PNAMP [Figs. 7(c) and 7(d)] and GCM [Figs. 7(e) and 7(f)] approaches we do not observe differences between the parametrizations either. In the former, shell effects are still present at $Z=20,28,40$, and 82 ; in the latter, the lead isotopes have the most prominent ones.

Because both PNAMP and GCM methods depend crucially on the intrinsic deformation, we also represent the correlation energy gains obtained for the different approaches as a function of the quadrupole deformation $\beta_{2}$ obtained at the HFB level 

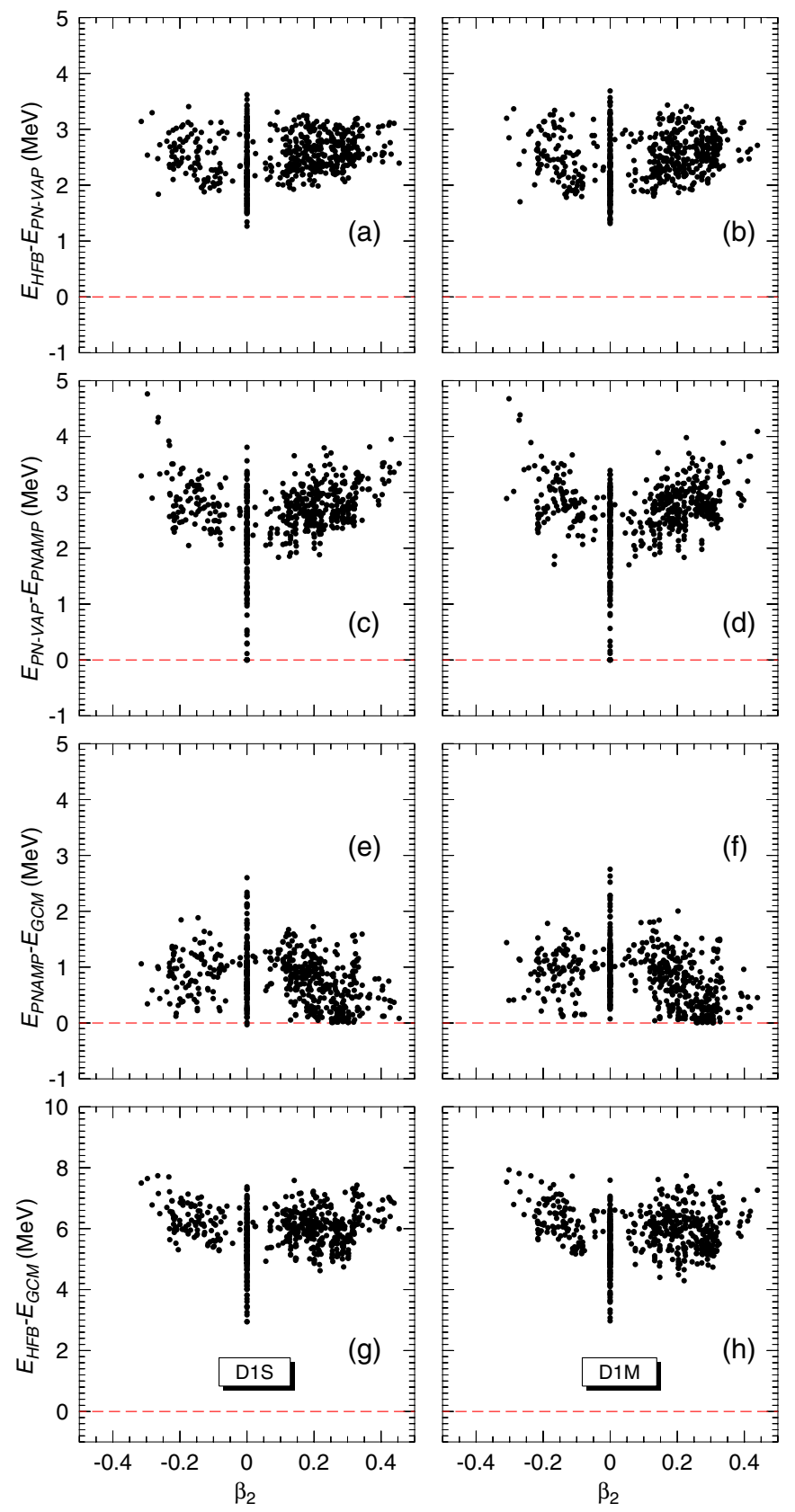

FIG. 8. (Color online) Same as Fig. 6, but as a function of the quadrupole deformation found at the MF-HFB-level.

for each nucleus (Fig. 8). In all cases, the largest spread in correlation energy gain is obtained in the spherical point $\beta_{2}=$ 0 . Hence, for this intrinsic deformation we observe energy gains ranging from $1.2-3.5,0.0-3.7$, and $0.0-2.8 \mathrm{MeV}$ for PN-VAP [Figs. 8(a) and 8(b)], PNAMP [Figs. 8(c) and 8(d)], and GCM [Figs. 8(e) and 8(f)], respectively. These spreads are smaller for the rest of the deformations. Additionally, the energy gain does not depend very much on the size and sign of the quadrupole deformation in both the PN-VAP [Figs. 8(a) and 8(b)] and the GCM [Figs. 8(e) and 8(f)] approaches. For the PNAMP case [Figs. 8(c) and 8(d)], a mild trend of having larger energy gains with increasing $\left|\beta_{2}\right|$ values is obtained. Similar results are obtained with Skyrme functionals (see Fig. 7 of

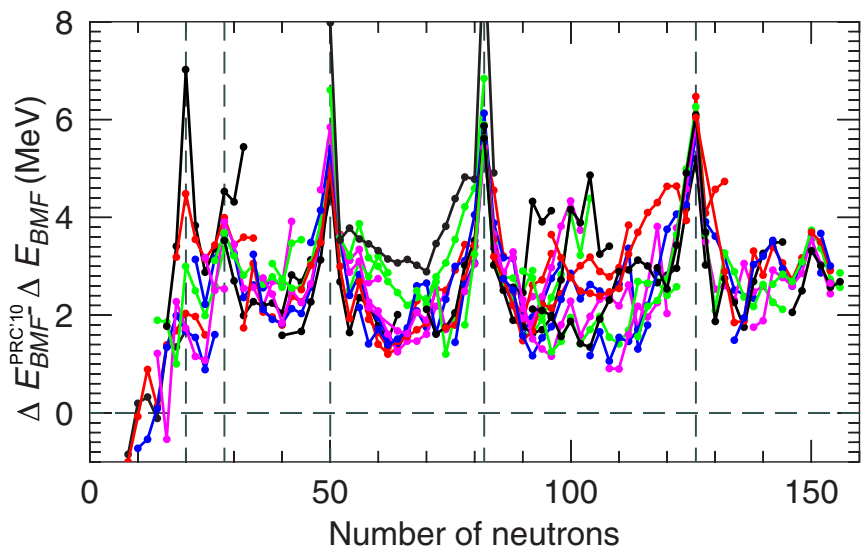

FIG. 9. (Color online) Difference between the total correlation energies given by the 5DCH model in Ref. [27] and the ones calculated with the present SCCM method. Isotopic chains are connected by lines with the same color and the interaction in all of the cases is Gogny D1S.

Ref. [25]), showing that these patterns depend on the method rather than on the choice of the functional.

To end this section, we also compare the total correlation energies obtained with the present axial SCCM calculations with those provided by the 5DCH for Gogny D1S given in the Supplemental Material of Ref. [27]. We observe in Fig. 9 that, except for a few light nuclei, the correlations given by the present method are about $2-3 \mathrm{MeV}$ larger than the $5 \mathrm{DCH}$ ones. Even larger differences are found around the shell closures because the 5DCH calculation gives positive correlation energies in those nuclei. As a matter of fact, such an anticorrelation energy is set to zero in Ref. [27]. Because the 5DCH method is based on a GOA, it is not variationally consistent with the underlying HFB functional. Furthermore, although the triaxial degree of freedom is included in the collective Hamiltonian, the present axial SCCM calculations include both symmetry restorations and GCM without GOA that produce larger correlation energies. These SCCM correlation energies are negative everywhere [see Fig. 6(g), for instance] and including other degrees of freedom such as the triaxiality will produce even more negative values. In addition, although convergence of both HFB and SCCM is not globally reached, we can at least determine where it has been achieved. Figure 9 shows that the difference in correlation energies between the present calculations and the 5DCH approach is rather constant along the whole nuclear chart, apart from the spikes around shell closures. This indicates that the 5DCH approach may suffer from similar convergence issues for heavy nuclei. However, one should keep in mind that, owing to its nonvariational nature, one cannot strictly speak of converged 5DCH calculations.

\section{B. Comparison with experimental data}

\section{Masses}

We now compare the results obtained with the different approximations with the experimental data extracted from the most recent AME [11]. In Fig. 10 the differences between the experimental and theoretical masses are plotted for the 

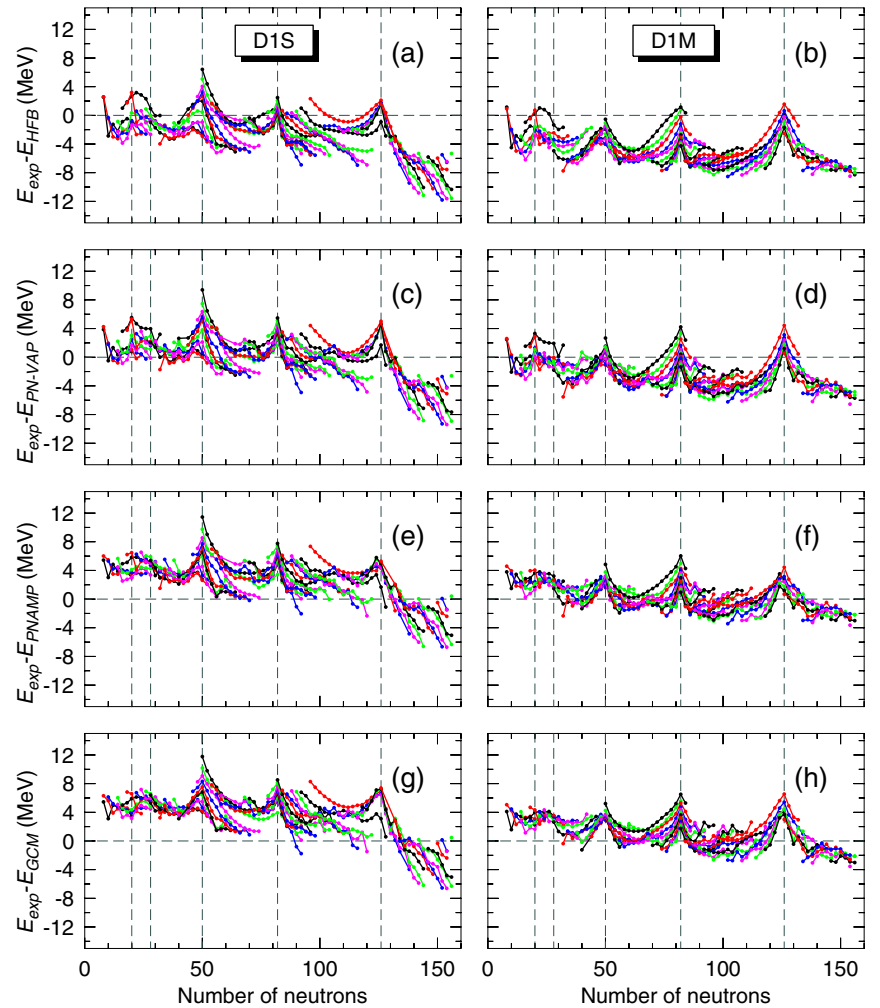

FIG. 10. (Color online) Difference between the experimental total energies (taken from Ref. [11]) and (a),(b) HFB; (c),(d) PN-VAP; (e),(f) PNAMP; (g),(h) GCM total energies calculated with the Gogny D1S (left column) and D1M (right column) parametrizations. Lines connect isotopic chains starting from $Z=10$. Black, red, blue, magenta, and green lines represent isotopic chains with $Z=$ $x 0, x 2, x 4, x 6$, and $x 8$, where $x=1,2, \ldots$, etc. Dashed vertical lines mark the neutron magic numbers 20, 28, 50, 82, and 126 .

different MF and BMF approaches. More quantitative results are written in Table II for the D1S and D1M parametrizations.

Starting from the oldest parametrization, i.e., D1S, we notice first its poor performance in describing experimental masses. In none of the many-body approaches studied here, the rms deviation is less than $2.6 \mathrm{MeV}$. This is explained by three major drawbacks of this parametrization (see left column of Fig. 10). The first one is the presence of residual shell

TABLE II. rms comparison between theoretical calculations and experimental data for total energies and two-neutron and two-proton separation energies. All energies are in $\mathrm{MeV}$.

\begin{tabular}{lccc}
\hline \hline D1S & $E$ & $S_{2 n}$ & $S_{2 p}$ \\
\hline HFB & 3.53 & 0.98 & 1.15 \\
PN-VAP & 2.62 & 1.10 & 1.11 \\
PNAMP & 3.75 & 0.98 & 1.00 \\
GCM & 4.45 & 0.95 & 1.00 \\
D1M & $E$ & $S_{2 n}$ & $S_{2 p}$ \\
HFB & 5.29 & 0.89 & 0.99 \\
PN-VAP & 3.14 & 1.03 & 0.96 \\
PNAMP & 1.79 & 0.89 & 0.86 \\
GCM & 2.17 & 0.85 & 0.87 \\
\hline \hline
\end{tabular}

effects. In all of the approaches, peaks at the neutron magic numbers $N=50,82$, and 126 are observed. As it discussed above, BMF energy gains are smaller in the shell closure nuclei [see Fig. 6(g)]. Therefore, these peaks are reduced when BMF effects are taken into account but the reduction is clearly insufficient to bring the theory closer to the experiment. The second drawback is the systematic drift towards less bound systems in nuclei with increasing neutron excess. The origin of the problem is in the symmetry energy provided by Gogny D1S. This parametrization does not reproduce the correct curvature in the neutron matter equation of state given by $a b$ initio approaches [22], producing a lack of binding energy in neutron-rich nuclei. Again, BMF effects do not change this trend. Nevertheless, the spread in light nuclei (from $N=10-40)$ found at the MF and PN-VAP approximations [Fig. 10(a)] is significantly reduced when PNAMP and GCM are taken into account [Figs. 10(e)-10(g)]. The third drawback is the way in which the parameters of the interaction were obtained. Hence, the parameters of the oldest realizations of the Gogny interaction were fitted to reproduce experimental data with the HFB method but leaving some room for eventual BMF effects [45]. However, some overbinding is still obtained with respect to the experimental values. The evolution of the rms values given in the second column of Table II reflects also this effect, obtaining for the most sophisticated many-body method used in this work a rms deviation of $4.45 \mathrm{MeV}$ (for 598 masses).

The D1M parametrization [9] was built to correct these shortcomings of the D1S by performing a fit to a large set of experimental masses using the 5DCH method [27] to include BMF effects. That led to a rms deviation from data of $\sim 0.798 \mathrm{MeV}$ (for 2149 masses). Except for the inclusion of triaxiality and the lack of quantum number projections, the $5 \mathrm{DCH}$ method can be considered as a GOA of the method used in this work [35]. Let us analyze now the performance of D1M in combination with the present axial SCCM method which does not assume such a GOA approximation. In the right column of Fig. 10 the difference between experimental and theoretical masses obtained with the D1M parametrization are shown. Here we observe that the drift and, partially, the overbinding found with the D1S parametrization are corrected. However, as stated in Ref. [9], strong shell effects are still present and the theoretical results that overestimate the binding energies around the magic neutron numbers, particularly at $N=50$, 82 , and 126 . This behavior is not corrected by including BMF correlations of the kind studied in this work. Nevertheless, the addition of correlations improves the agreement with data with respect to the MF results. Because the D1M parameters were fitted taking already into account BMF effects, the results at the HFB level are underbound with respect to the experimental values [Fig. 10(b)]. A very large rms deviation is obtained for this approach and a much smaller deviation is obtained for the rest (see Table II). However, the correlation energies attained by the GCM are larger than the ones provided by the $5 \mathrm{DCH}$, as discussed above. This produces an excess of total energy also with this parametrization when the axial shape mixing with quantum number projection is taken into account [Fig. 10(h)]. The rms value for the GCM approach with the D1M parametrization is 2.17 (for 598 masses). 

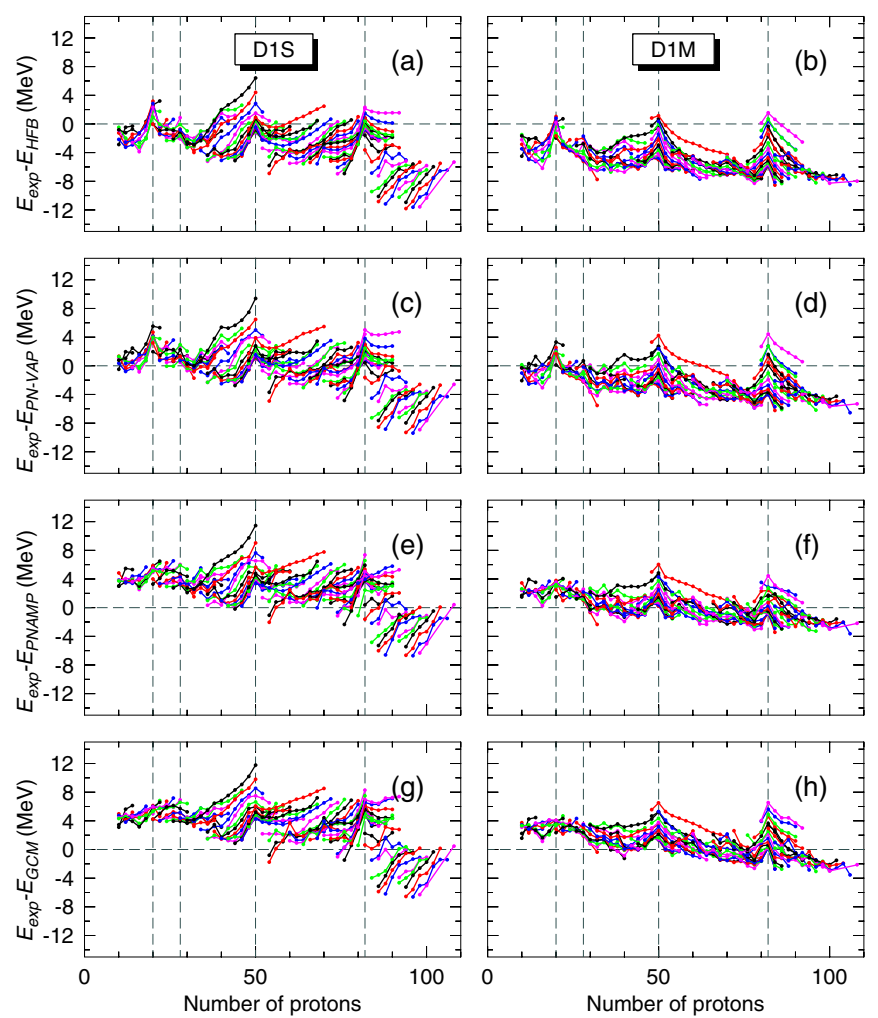

FIG. 11. (Color online) Same as Fig. 10 but as a function of the number of protons. Lines connect isotonic chains starting from $N=$ 10. Black, red, blue, magenta, and green lines represent isotopic chains with $N=x 0, x 2, x 4, x 6$, and $x 8$, where $x=1,2, \ldots$, etc. Dashed vertical lines mark the neutron magic numbers 20, 28, 50, and 82 .

It is worth mentioning that the global rms values themselves do not provide the complete picture of the comparison with the experimental data because some compensation effects can occur in different regions of the nuclear chart. A rms value independent of the number of particles - including BMF effects - must be pursued even though an excess of total energy would be expected when BMF corrections are incorporated on top of a functional fitted to reproduce data with less correlated ground states. In this sense, BMF corrections work better in reducing the spread and shell gaps in $N \leqslant 28$ nuclei than in heavier systems (see Fig. 10), although, from the point of view of the rms, light nuclei are farther away from the experiment than the heavy ones.

To check the possible shell effects appearing in isotonic chains, we represent in Fig. 11 the energy differences with the experimental masses obtained for the different many-body approaches and parametrizations as a function of the number of protons. These shell effects, though still present, are slightly less pronounced than in the isotopic chains. This is in agreement with the results found with Skyrme interactions [25]. However, it is interesting to note that the relative overbinding found at $Z=20$ at the HFB level is smoothened out with BMF approaches in both parametrizations while $Z=50$ and 82 persist. Finally, we observe a clear difference between the results provided by the two parametrizations. For D1M rather symmetric energy differences around the shell closures are found while for D1S larger energies are obtained for larger values of $Z$ within a given isotonic chain. Because the isotonic chains start normally with $N>Z$, this behavior reflects again a lack of ground-state energy provided by the D1S parametrization in nuclei with neutron excess, i.e., the symmetry energy problem already mentioned.

To conclude this section, we can state that both the symmetry energy problem and the overbinding produced by the inclusion of BMF effects can be solved by modifying the parametrization, as almost done with the introduction of the Gogny D1M interaction. However, the energy excess obtained in the magic nuclei (relative to the energy predicted in the midshell nuclei), although reduced, is not washed out by taking into account the present BMF effects. It is still an open question whether the current BMF functionals, with parameters self-consistently fitted and probably extended to include other collective degrees of freedom and symmetry restorations, are able to produce flat energy differences instead of the parabolic behavior found in Figs. 10 and 11 (and in Refs. [9,22,25,27]). These tasks are highly demanding, both the refit of the interaction and the inclusion of, for example, pairing fluctuations [54], triaxial [38,55-57] and/or octupole $[58,59]$ shapes with the corresponding symmetry restorations and configuration mixing. However, it is possible that the central, spin-orbit, and density-dependent terms of the starting Gogny interaction have to be modified, including, for instance, explicit tensor terms [60,61].

Finally, because the present calculations are not fully converged, no other degrees of freedom are taken into account - triaxiality, octupolarity, etc.-rms values for D1S and/or D1M parametrizations given in Table II should be considered as a qualitative description of the effect on the masses produced by the different approaches rather than the final values.

\section{Two-particle separation energies and shell gaps}

Most of the time, the relevant quantities for calculating reaction rates, $Q$ values, etc., with astrophysical interest are not the absolute energies shown in the previous section but energy differences between those masses. We analyze now its performance on two-nucleon separation energies $\left(S_{2 n}, S_{2 p}\right)$, because the present GCM method with the Gogny functional is not well developed for computing odd nuclei yet:

$$
\begin{aligned}
& S_{2 n}(Z, N)=E(Z, N-2)-E(Z, N), \\
& S_{2 p}(Z, N)=E(Z-2, N)-E(Z, N) .
\end{aligned}
$$

These quantities are plotted in Figs. 12 and 13 and analyzed quantitatively in Table II. The overall behavior of the experimental values is quite well reproduced. The rms deviations from experimental values for $S_{2 n}$ and $S_{2 p}$ are much better than in the masses for both parametrizations. However, we observe important local differences between the experimental data and theory. On the one hand, the experimental curves are much smoother, having always for a constant number of protons (neutrons) a continuous decrease in the $S_{2 n}\left(S_{2 p}\right)$ when adding neutrons (protons). This is not the case for the theoretical data, where jumps and crossings between isotonic (isotopic) chains in the $S_{2 n}\left(S_{2 p}\right)$ separation energies are shown. This drawback 

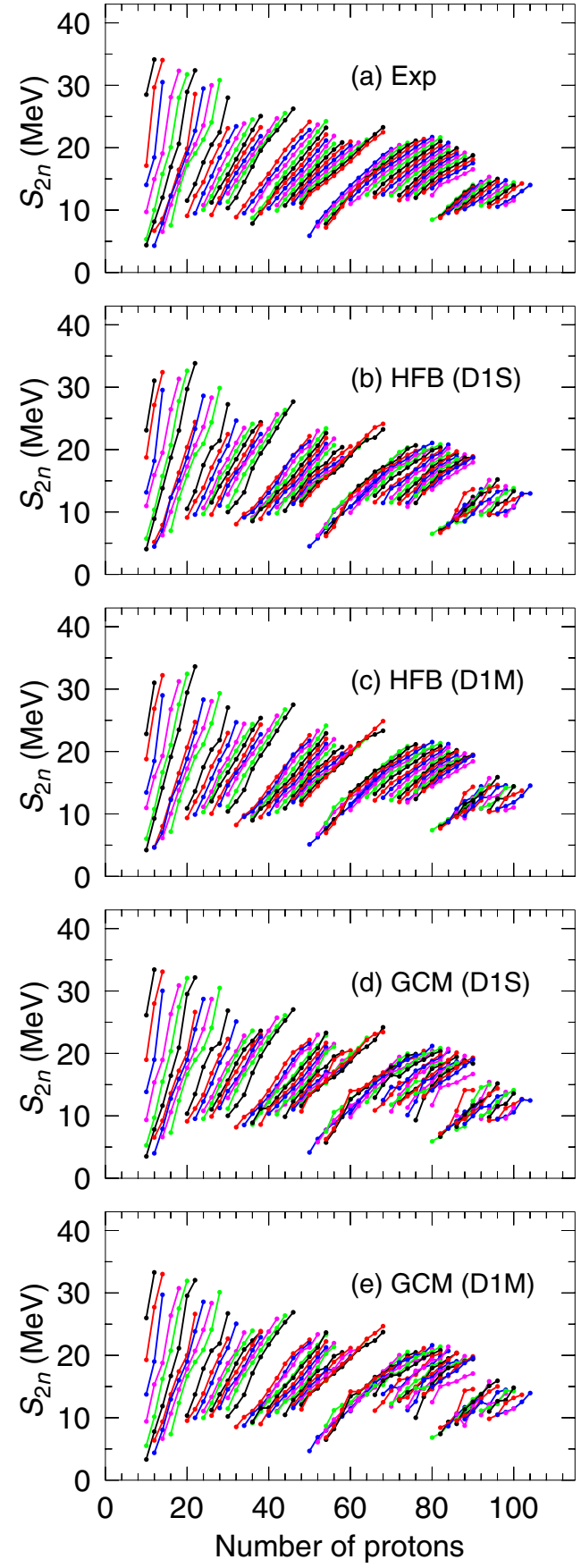

FIG. 12. (Color online) Two-neutron separation energies as a function of the number of protons: (a) experimental data (taken from Ref. [11]); (b) HFB; (d) GCM calculated with the Gogny D1S parametrization and (c) HFB; (e) GCM calculated with D1M parametrization. Lines connect isotonic chains starting from $N=10$. Black, red, blue, magenta, and green lines represent isotopic chains with $N=x 0, x 2, x 4, x 6$, and $x 8$, where $x=1,2, \ldots$, etc.

is obtained all over the nuclear chart independently of the parametrization and is a bit larger in the GCM approach than in the HFB result. Its origin could be related to a convergence problem discussed in Sec. II and/or the lack of other degrees of freedom such as triaxiality or octupolarity.
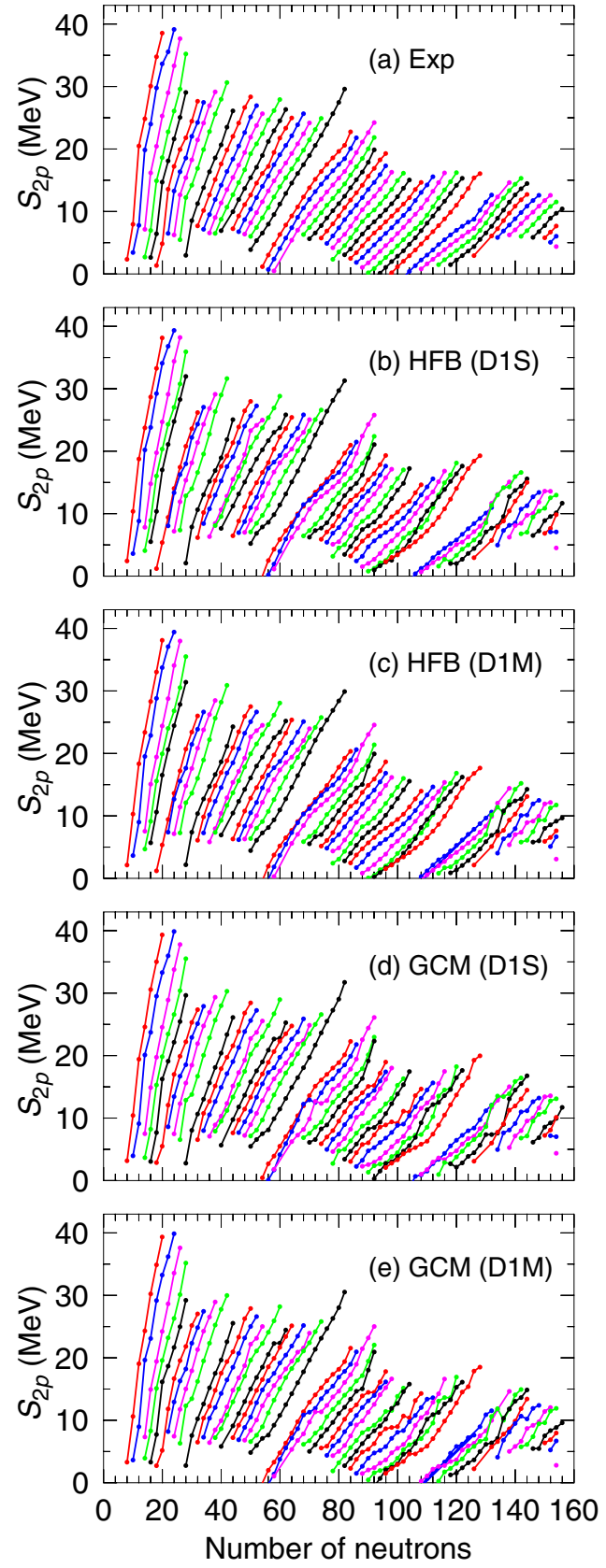

FIG. 13. (Color online) Two-proton separation energies as a function of the number of neutrons: (a) Experimental data (taken from Ref. [11]); (b) HFB; (c) GCM calculated with the Gogny D1S parametrization. Lines connect isotopic chains starting from $Z=12$. Black, red, blue, magenta and green lines represent isotopic chains with $Z=x 0, x 2, x 4, x 6$, and $x 8$, where $x=1,2, \ldots$, etc.

Finally, we analyze the neutron and proton shell gaps associated to magic numbers by zooming in the $S_{2 n}$ energies for $N=20,22,28,30,50,52,82,84,126$, and 128 isotonic chains and the $S_{2 p}$ energies for $Z=20,22,28$, 30, 50, 52, 82, and 84 isotopic chains in Figs. 14 and 15. We observe first that the different parametrizations (D1S and D1M) provide for this set of nuclei very similar results. Furthermore, the MF approach tends to predict larger shell 

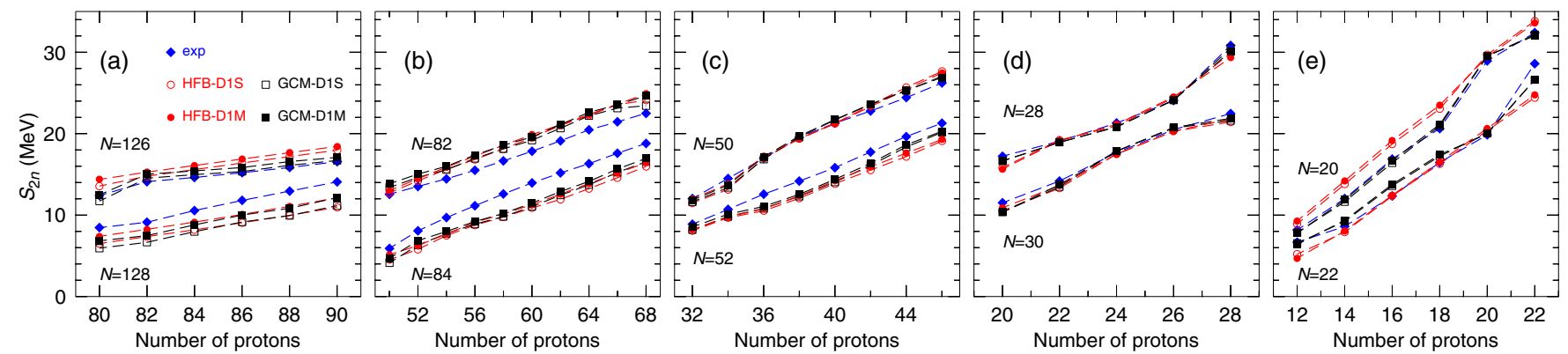

FIG. 14. (Color online) Two-neutron separation energies for (a) $N=126,128$; (b) $N=82,84$; (c) $N=50,52$; (d) $N=28,30$; and (e) $N=20,22$ isotonic chains. Blue diamonds, red circles, and black boxes represent the experimental, MF, and GCM results, respectively. Open (solid) symbols are calculated with the Gogny D1S (D1M) parametrization.

gaps than the experimental ones, except for $N, Z=28$ magic numbers, where the agreement with the experimental data is very good. Correlations beyond the static MF tend to reduce these gaps, almost matching the experimental results in $N, Z=20$ and $Z=50$. However, the reduction is not enough to reproduce the actual gaps in $N=50,82$, and 126 nor $Z=82$. Therefore, the shell quenching obtained by including BMF effects reported by Bender et al. in Ref. [26] and by Delaroche et al. in Ref. [27] is only partially reproduced here. In addition, the latter results are much smoother than the results shown in Figs. 12 and 13. As mentioned above (see Fig. 9), the amount of BMF correlation energies obtained by the present SCCM method is larger than the one obtained by solving the $5 \mathrm{DCH}$ model, even though in the latter the triaxiality is included. Furthermore, calculations reported in Refs. [25,26] are very similar to the ones presented here, except for the use of GOA (and topGOA) approximations. Therefore, the smoothness of the $S_{2 n}\left(S_{2 p}\right)$ separation energies in such calculations could be related to the GOA approximations used in those references. Nevertheless, a better convergence and the addition of other degrees of freedom are needed to check whether the present interactions can reproduce the smoothness of the experimental data.

\section{3. $2_{1}^{+}$excitation energies}

We finally compare the $2_{1}^{+}$excitation energies obtained with our present GCM global calculations with the experimental data compiled in Ref. [62]. It is important to note again that the convergence of the results is not fully guaranteed, especially in heavy nuclei where a working basis including 11 major oscillator shells could be too small (see Sec. II and Fig. 5, bottom row). Therefore, the values obtained with this reduced space must be taken with caution. In any case, we consider these results relevant to extract a global performance of the method, and, in particular, to compare the results provided by the two Gogny parametrizations for this observable. We can also compare with the results of similar studies already reported with the Skyrme SLy4 [63] (with a topological GOA in the angular-momentum projection and a limited number of intrinsic states in the GCM) and Gogny D1S [27,64] within the $5 \mathrm{DCH}$ framework. However, in the latter cases an educated selection of nuclei where the method is better suited was made and sets of 359 [63], 519 [64], and 513 [27] nuclei were chosen in these papers.

Both the experimental values and the results of the GCM calculations for the $2_{1}^{+}$energies are shown in Fig. 16. We observe clearly an enhancement of the excitation energies at the proton and neutron magic numbers. Additionally, the $2_{1}^{+}$excitation energies corresponding to $N=20,28,50,82$, and 126 isotonic chains and $\mathrm{Ca}, \mathrm{Ni}, \mathrm{Sn}$, and $\mathrm{Pb}$ isotopes are clearly above the rest both experimentally and in the calculations. However, we see that the two parametrizations provide almost identical results for this observable even though they behave very differently for masses. The global behavior of the experimental data is well reproduced, although the
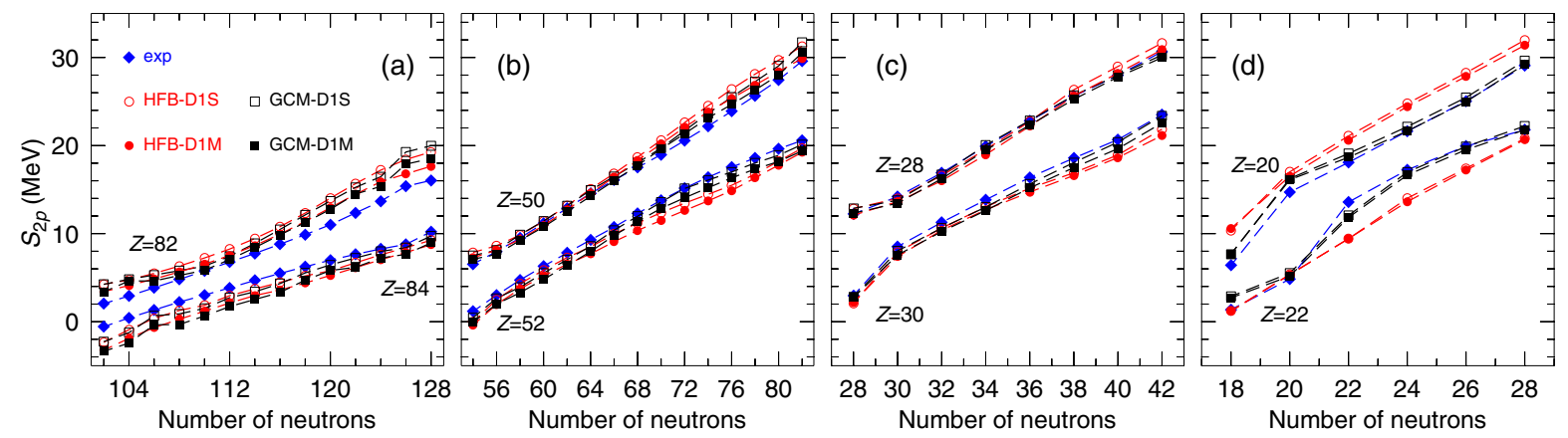

FIG. 15. (Color online) Two-proton separation energies for (a) $Z=82,84$; (b) $Z=50,52$; (c) $Z=28,30$; and (d) $N=20,22$ isotopic chains. Blue diamonds, red circles, and black boxes represent the experimental, MF, and GCM results, respectively. Open (solid) symbols are calculated with the Gogny D1S (D1M) parametrization. 

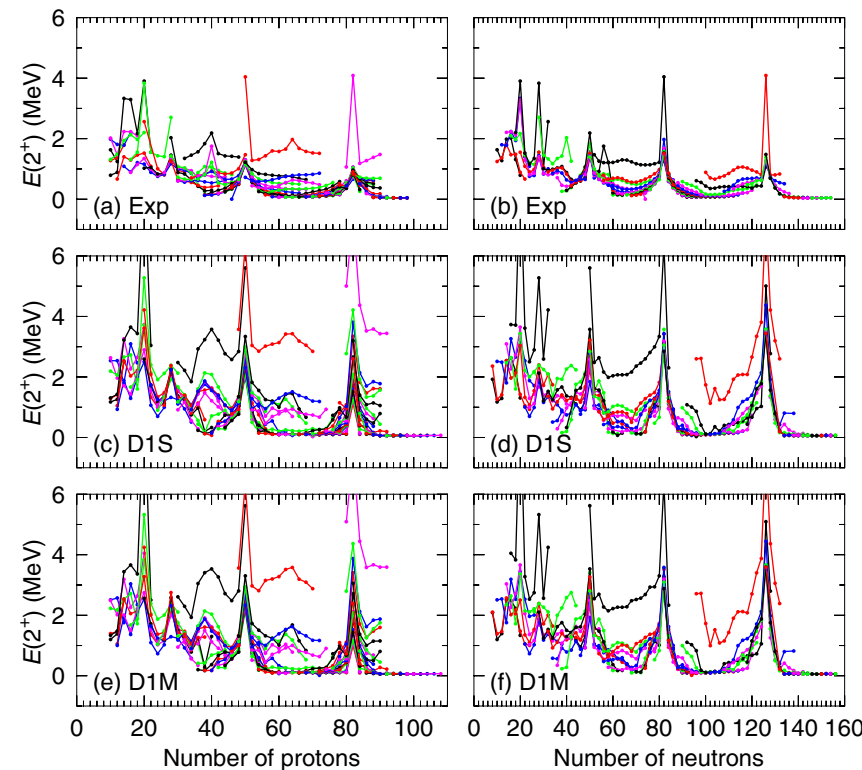

FIG. 16. (Color online) $2_{1}^{+}$excitation energies for (a),(b) experimental data (taken from Ref. [62]); (c),(d) GCM-Gogny D1S; and (e),(f) GCM-Gogny D1M. Lines connect isotonic chains starting from $N=10$ (left column) and isotopic chains starting from $Z=10$ (right column). The color code is the same as in previous figures.

calculations show a less smooth behavior and a systematic overestimation of the experimental values.

The latter drawback can be better seen in Figs. 17(a) and 17(b), where the theoretical values versus the experimental energies are represented for D1S and D1M parametrizations, respectively. Although we observe a clear correlation between the two quantities, the theoretical predictions tend to overestimate the empirical values. We do not find significant differences between the D1S and D1M parametrizations. To better estimate quantitatively the differences with the experimental results, we follow the analysis performed in Refs. $[27,63,64]$. In these works, the so-called logarithmic error- $R_{E}$-and its standard deviation- $\sigma_{E}$-from the average $-\bar{R}_{E}$-are defined as

$$
\begin{aligned}
R_{E} & =\ln \left[E\left(2_{1}^{+}\right)_{\mathrm{th}} / E\left(2_{1}^{+}\right)_{\exp }\right], \\
\sigma_{E} & =\left\langle\left(R_{E}-\bar{R}_{E}\right)^{2}\right\rangle^{1 / 2} .
\end{aligned}
$$

In Figs. 17(c) and 17(d) we show histograms representing the number of nuclei with a given value of $R_{E}$ for D1S and D1M parametrizations. We find rather symmetric distributions with mean values and standard deviations of $\bar{R}_{E}=0.32(0.35)$ and $\sigma_{E}=0.42(0.43)$, respectively, for D1S (D1M). Similar results are obtained with Skyrme SLy4 [63] with a more restricted set of nuclei (see Table III). This systematic drift towards larger values is a consequence of the variational method. In this framework, the ground-state $0_{1}^{+}$are favored with respect to other states, pulling down its energy and stretching the final spectra. The inclusion of additional degrees of freedom such as triaxiality, time-reversal symmetry-breaking, and quasiparticle excitations, which are more relevant in the excited states than in the ground state,
TABLE III. Logarithmic errors and deviations for the $2_{1}^{+}$excitation energies computed for GCM-D1S, GCM-D1M, GCM-SLy4 [63],

\begin{tabular}{|c|c|c|c|c|c|}
\hline & $\begin{array}{c}\text { GCM-D1S } \\
(534)\end{array}$ & $\begin{array}{c}\text { GCM-D1M } \\
(534)\end{array}$ & $\begin{array}{c}\text { GCM-SLy4 } \\
\text { (359) }\end{array}$ & $\begin{array}{c}\text { 5DCH-D1S } \\
(513)\end{array}$ & $\begin{array}{c}\text { 5DCH-D1S } \\
(519)\end{array}$ \\
\hline $\bar{R}_{E}$ & 0.32 & 0.35 & 0.51 & 0.11 & 0.12 \\
\hline$\sigma_{E}$ & 0.42 & 0.43 & 0.38 & 0.35 & 0.33 \\
\hline
\end{tabular}
and 5DCH-D1S [27,64]. Experimental data are taken from Ref. [62].

allows a variational description of these excited states and the excitation energies can be reduced. For example, local studies with a GCM method including triaxial angular momentum projection [38,55-57] or pairing fluctuations [54] have already shown this effect. However, quantum number projections and GCM can modify significantly both the equilibrium deformation of the system $\left({ }^{32} \mathrm{Mg}\right.$ is the paradigm for this effect [37]) and the collective masses. Because these parametrizations are not fitted with using the BMF many-body states of this work, the MF and BMF collective behavior can be different. For instance, angular-momentum projection could overestimate a quadrupole deformation that could be correct at the HFB level. Therefore, the excitation energies should be taken into account in a future refit of the interaction.

We finally comment on the smaller deviation and dispersion obtained with Gogny D1S using the 5DCH method [27] shown in Table III. In this case, the inclusion of the triaxial degree of freedom and the fit of the inertia parameters with a cranking approximation can improve the description of the $2_{1}^{+}$excitation energies. However, because 5DCH does not include either particle-number or angular-momentum projection, spurious contributions of MF states with the wrong quantum numbers
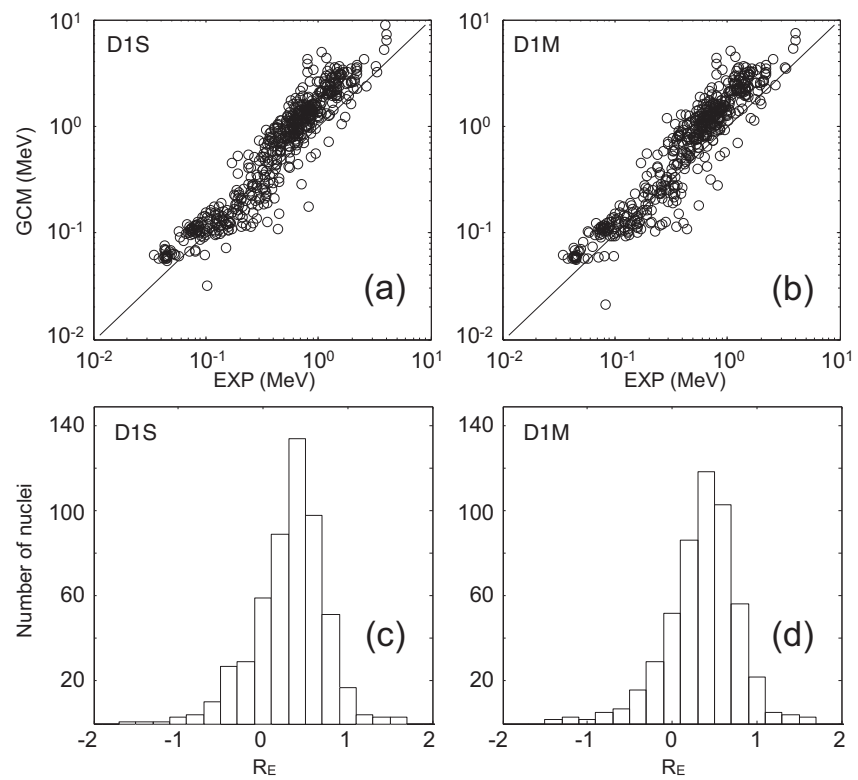

FIG. 17. GCM versus experimental $2_{1}^{+}$excitation energies for (a) D1S and (b) D1M parametrizations. (c),(d) Histograms of the logarithmic errors of the $2_{1}^{+}$states. 
can produce a collapse of the excited states [54]. In any case, further analyses, beyond the scope of this work, are needed to compare the 5DCH approach with a full SCCM approximation.

\section{SUMMARY AND OUTLOOK}

In this work, correlation energies, total energies, twoneutron separation energies, and $2_{1}^{+}$excitation energies for a large set of even-even nuclei along the nuclear chart have been presented. The theoretical results have been obtained by using self-consistent MF (HFB) and BMF methods, including particle-number and angular-momentum restorations and axial quadrupole shape mixing without GOAs. The underlying interaction used in all of the calculations was Gogny with two different parametrizations, D1S and D1M. The convergence of the results as a function of the number of harmonic oscillator shells included in the basis has been analyzed. Hence, the convergence of the total energies are not fully guaranteed and extrapolation schemes to infinite bases should be implemented in the near future. Nevertheless, a large number of harmonic oscillator shells, $N_{\text {s.h.o. }}=19$, has been used for the HFB part, showing a better performance than the usual rule of having a number of single-particle states equal to eight times the larger number among the protons and neutrons of a given nucleus. Additionally, the calculated BMF correlations and particle separation energies are less dependent on the number of harmonic oscillator shells than the total energies. Concerning those BMF correlations, both parametrizations, D1S and D1M, give similar correlation energies with respect to their $\mathrm{MF}$ solutions.

Compared to the experimental data, the D1S parametrization shows a symmetry energy problem which produces a lack of binding energy in neutron-rich systems. This fact is reflected in a drift in the difference between theoretical and experimental energies for nuclei with a neutron excess which is corrected in the D1M parametrization. However, strong shell effects (stronger as a function of the neutron number) are still present in both realizations and parabolic instead of flat energy differences are found between two consecutive magic numbers. BMF correlations tend to reduce these differences because rotational and vibrational corrections are larger in the midshell than in closed-shell nuclei, but this reduction is not sufficient to remove the difference between the experimental and theoretical values.

Energy differences such as two-neutron separation energies are in a better agreement with experimental data but still not satisfactory. Hence, BMF correlations bring the theoretical predictions towards the experimental values for $N, Z=20$ shell gaps. However, some problems like artificial jumps, crossing, and overestimation of the shell gaps for heavier nuclei are found in the calculations and they are not fully corrected by including BMF effects.

Additionally, we have reported the results for $2_{1}^{+}$excitation energies calculated with $N_{\text {s.h.o. }}=11$ shells. We have obtained similar results for D1S and D1M parametrizations, having a systematic stretching of the $2_{1}^{+}$energies which is more related to the method used to solve the many-body problem rather than the parametrization itself.

As an outlook, some improvements should be taken into account in the near future, in particular:

(i) convergence of the results with the properties of the harmonic oscillator working basis;

(ii) triaxial and other degrees of freedom should be included explicitly in the calculations.

In the long term, a new parametrization of the Gogny interaction (or any other type of EDF) including in the fitting procedure fully converged SCCM corrections with triaxial and octupolar states is desirable.

Finally, new experimental data would be very helpful to constrain further the current and future models.

\section{ACKNOWLEDGMENTS}

We acknowledge the support from GSI-Darmstadt and CSC-Loewe-Frankfurt computing facilities. This work was supported by the BMBF-Verbundforschungsprojekt Grant No. 06DA7047I, the Ministerio de Economía y Competitividad through the Programa Ramón y Cajal 2012 Grant No. 11420, and the Helmholtz Association through the Nuclear Astrophysics Virtual Institute (Grant No. VH-VI-417).
[1] K. Blaum, Phys. Rep. 425, 1 (2006).

[2] P. Möller, J. R. Nix, W. D. Myers, and W. J. Swiatecki, At. Data Nucl. Data Tables 59, 185 (1995).

[3] P. Möller, W. D. Myers, H. Sagawa, and S. Yoshida, Phys. Rev. Lett. 108, 052501 (2012).

[4] N. Wang, M. Liu, and X. Wu, Phys. Rev. C 81, 044322 (2010).

[5] N. Wang, M. Liu, X. Wu, and J. Meng, Phys. Lett. B 734, 215 (2014).

[6] J. Duflo and A. P. Zuker, Phys. Rev. C 52, R23 (1995).

[7] S. Goriely, N. Chamel, and J. M. Pearson, Phys. Rev. C 88, 024308 (2013).

[8] S. Goriely, N. Chamel, and J. M. Pearson, Phys. Rev. C 88, 061302(R) (2013).
[9] S. Goriely, S. Hilaire, M. Girod, and S. Péru, Phys. Rev. Lett. 102, 242501 (2009).

[10] A. H. Wapstra, G. Audi, and C. Thibault, Nucl. Phys. A 729, 129 (2003).

[11] M. Wang, G. Audi, A. H. Wapstra, F. G. Kondev, M. MacCormick, X. Xu, and B. Pfeiffer, Chin. Phys. C 36, 1603 (2012).

[12] A. Arcones and G. Martínez-Pinedo, Phys. Rev. C 83, 045809 (2011).

[13] S. Brett, I. Bentley, N. Paul, R. Surman, and A. Aprahamian, Eur. Phys. J. A 48, 184 (2012).

[14] R. Roth, J. Langhammer, A. Calci, S. Binder, and P. Navratil, Phys. Rev. Lett. 107, 072501 (2011). 
[15] D. J. Dean and M. Hjorth-Jensen, Phys. Rev. C 69, 054320 (2004).

[16] H. Hergert, S. Binder, A. Calci, J. Langhammer, and R. Roth, Phys. Rev. Lett. 110, 242501 (2013).

[17] V. Soma, C. Barbieri, and T. Duguet, Phys. Rev. C 87, 011303 (2013).

[18] T. Otsuka, T. Suzuki, J. D. Holt, A. Schwenk, and Y. Akaishi, Phys. Rev. Lett. 105, 032501 (2010).

[19] D. Steppenbeck et al., Nature (London) 502, 207 (2013).

[20] J. D. Holt, J. Menéndez, and A. Schwenk, Phys. Rev. Lett. 110, 022502 (2013).

[21] N. Chamel, S. Goriely, and J. M. Pearson, Nucl. Phys. A 812, 72 (2008).

[22] F. Chappert, M. Girod, and S. Hilaire, Phys. Lett. B 668, 420 (2008).

[23] S. Goriely, M. Samyn, and J. M. Pearson, Phys. Rev. C 75, 064312 (2007).

[24] M. Bender, P.-H. Heenen, and P.-G. Reinhard, Rev. Mod. Phys. 75, 121 (2003).

[25] M. Bender, G. F. Bertsch, and P.-H. Heenen, Phys. Rev. C 73, 034322 (2006).

[26] M. Bender, G. F. Bertsch, and P.-H. Heenen, Phys. Rev. C 78, 054312 (2008).

[27] J.-P. Delaroche, M. Girod, J. Libert, H. Goutte, S. Hilaire, S. Péru, N. Pillet, and G. F. Bertsch, Phys. Rev. C 81, 014303 (2010).

[28] K. Q. Lu, Z. X. Li, Z. P. Li, J. M. Yao, and J. Meng, Phys. Rev. C 91, 027304 (2015).

[29] J.-F. Berger, M. Girod, and D. Gogny, Nucl. Phys. A 428, $23 \mathrm{c}$ (1984).

[30] J.-F. Berger, M. Girod, and D. Gogny, Comput. Phys. Commun. 63, 365 (1991).

[31] B. Bally, B. Avez, M. Bender, and P.-H. Heenen, Phys. Rev. Lett. 113, 162501 (2014).

[32] P. Möller, R. Bengtsson, B. G. Carlsson, P. Olivius, and T. Ichikawa, Phys. Rev. Lett. 97, 162502 (2006).

[33] L. M. Robledo and G. F. Bertsch, Phys. Rev. C 84, 054302 (2011).

[34] L. M. Robledo, J. Phys. G: Nucl. Part. Phys. 42, 055109 (2015).

[35] P. Ring and P. Schuck, The Nuclear Many-body Problem (Springer-Verlag, Berlin, 1980).

[36] D. Lacroix, T. Duguet, and M. Bender, Phys. Rev. C 79, 044318 (2009).

[37] R. Rodríguez-Guzmán, J. L. Egido, and L. M. Robledo, Nucl. Phys. A 709, 201 (2002).

[38] T. R. Rodríguez and J. L. Egido, Phys. Rev. C 81, 064323 (2010).
[39] Although the working basis is spherical, the HFB states, defined by the transformation given in Eq. (3), can be deformed.

[40] M. Anguiano, J. L. Egido, and L. M. Robledo, Nucl. Phys. A 696, 467 (2001).

[41] L. M. Robledo, HFBAXIAL code, Universidad Autónoma de Madrid, 2002.

[42] T. R. Rodríguez and J. L. Egido, Phys. Rev. Lett. 99, 062501 (2007).

[43] https://wiki.gsi.de/cgi-bin/view/Linux/GridEngine.

[44] http://csc.uni-frankfurt.de/index.php?id=51\&L=0.

[45] J. Dechargé and D. Gogny, Phys. Rev. C 21, 1568 (1980).

[46] J. Decharge and L. Sips, Nucl. Phys. A 407, 1 (1983).

[47] S. Hilaire and M. Girod, Eur. Phys. J. A 33, 237 (2007).

[48] R. J. Furnstahl, G. Hagen, and T. Papenbrock, Phys. Rev. C 86, 031301 (2012).

[49] S. N. More, A. Ekström, R. J. Furnstahl, G. Hagen, and T. Papenbrock, Phys. Rev. C 87, 044326 (2013).

[50] R. J. Furnstahl, S. N. More, and T. Papenbrock, Phys. Rev. C 89, 044301 (2014).

[51] S. König, S. K. Bogner, R. J. Furnstahl, S. N. More, and T. Papenbrock, Phys. Rev. C 90, 064007 (2014).

[52] A. Arzhanov, T. R. Rodríguez, and G. Martínez-Pinedo (unpublished).

[53] J. E. García-Ramos and K. Heyde, Phys. Rev. C 89, 014306 (2014).

[54] N. L. Vaquero, J. L. Egido, and T. R. Rodríguez, Phys. Rev. C 88, 064311 (2013).

[55] M. Bender and P.-H. Heenen, Phys. Rev. C 78, 024309 (2008).

[56] J. M. Yao, J. Meng, P. Ring, and D. Vretenar, Phys. Rev. C 81, 044311 (2010).

[57] T. R. Rodríguez, Phys. Rev. C 90, 034306 (2014).

[58] P. A. Butler and W. Nazarewicz, Rev. Mod. Phys. 68, 349 (1996).

[59] E. Garrote, J. L. Egido, and L. M. Robledo, Phys. Rev. Lett. 80, 4398 (1998).

[60] T. Otsuka, T. Matsuo, and D. Abe, Phys. Rev. Lett. 97, 162501 (2006).

[61] M. Anguiano, M. Grasso, G. Co, V. De Donno, and A. M. Lallena, Phys. Rev. C 86, 054302 (2012).

[62] S. Raman, C. W. Nestor, and P. Tikkanen, At. Data Nucl. Data Tables 78, 1 (2001).

[63] B. Sabbey, M. Bender, G. F. Bertsch, and P.-H. Heenen, Phys. Rev. C 75, 044305 (2007).

[64] G. F. Bertsch, M. Girod, S. Hilaire, J.-P. Delaroche, H. Goutte, and S. Péru, Phys. Rev. Lett. 99, 032502 (2007). 